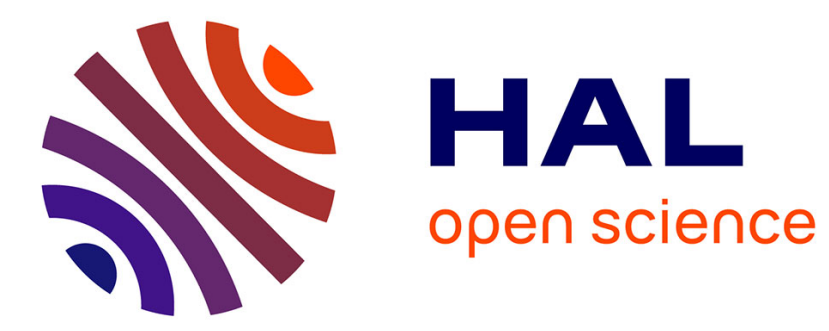

\title{
Dynamic Programming for Graphs on Surfaces
}

Juanjo Rué, Ignasi Sau, Dimitrios M. Thilikos

\section{To cite this version:}

Juanjo Rué, Ignasi Sau, Dimitrios M. Thilikos. Dynamic Programming for Graphs on Surfaces. ACM

Transactions on Algorithms, 2014, 10 (2), pp.1-26/8. 10.1145/2556952 . lirmm-01083690

\section{HAL Id: lirmm-01083690 \\ https://hal-lirmm.ccsd.cnrs.fr/lirmm-01083690}

Submitted on 17 Nov 2014

HAL is a multi-disciplinary open access archive for the deposit and dissemination of scientific research documents, whether they are published or not. The documents may come from teaching and research institutions in France or abroad, or from public or private research centers.
L'archive ouverte pluridisciplinaire HAL, est destinée au dépôt et à la diffusion de documents scientifiques de niveau recherche, publiés ou non, émanant des établissements d'enseignement et de recherche français ou étrangers, des laboratoires publics ou privés. 


\title{
Dynamic Programming for Graphs on Surfaces
}

\author{
JUANJO RUÉ, ICM \\ IGNASI SAU, CNRS-LIRMM \\ DIMITRIOS M. THILIKOS, National and Kapodistrian University of Athens
}

\begin{abstract}
We provide a framework for the design and analysis of dynamic programming algorithms for surface-embedded graphs on $n$ vertices and branchwidth at most $k$. Our technique applies to general families of problems where standard dynamic programming runs in $2^{O(k \cdot \log k)} \cdot n$ steps. Our approach combines tools from topological graph theory and analytic combinatorics. In particular, we introduce a new type of branch decomposition called surface cut decomposition, generalizing sphere cut decompositions of planar graphs which has nice combinatorial properties. Namely, the number of partial solutions that can be arranged on a surface cut decomposition can be upper-bounded by the number of non-crossing partitions on surfaces with boundary. It follows that partial solutions can be represented by a single-exponential (in the branchwidth $k$ ) number of configurations. This proves that, when applied on surface cut decompositions, dynamic programming runs in $2^{O(k)} \cdot n$ steps. That way, we considerably extend the class of problems that can be solved in running times with a single-exponential dependence on branchwidth and unify/improve most previous results in this direction.
\end{abstract}

Categories and Subject Descriptors: G.2.2 [Graph theory]: Graph Algorithms

General Terms: Algorithms; Theory.

Additional Key Words and Phrases: analysis of algorithms; parameterized algorithms; graphs on surfaces; branchwidth; graphs on surfaces; dynamic programming; polyhedral embeddings; non-crossing partitions.

ACM Reference Format:

Rué J., Sau I., Thilikos D.M. 2012. Dynamic Programming for Graphs on Surfaces. ACM Trans. Algor. -, -, Article - ( $-), 22$ pages.

DOI $=10.1145 / 0000000.0000000$ http://doi.acm.org/10.1145/0000000.0000000

\section{INTRODUCTION}

One of the most important parameters in the design and analysis of graph algorithms is the branchwidth of a graph. Branchwidth, together with its twin parameter of treewidth, can be seen as a measure of the resemblance of a graph to a tree. Its algorithmic importance dates back to the celebrated theorem of Courcelle (see e.g. [Courcelle 1988]), stating that graph problems expressible in Monadic Second Order Logic can be solved in $f($ bw $) \cdot n$ steps (here bw is the branchwidth ${ }^{1}$ and $n$ is the number of vertices of the input graph). Using parameterized complexity terminology, this implies that a large number of graph problems are fixed-parameter tractable when parameterized by the branchwidth of their input graph. As the bounds for $f(\mathbf{b w})$ provided by Courcelle's theorem are huge, the design of tailor-made dynamic programming algorithms for specific problems so that $f(\mathbf{b w})$ is a simple - preferably a single-exponential - function, became a natural (and unavoidable) ingredient for many results on graph algorithms (see [Arnborg 1985; Bodlaender 1988; Telle and Proskurowski 1997;

\footnotetext{
${ }^{1}$ The original statement of Courcelle's theorem used the parameter of treewidth instead of branchwidth. The two parameters are equivalent, in the sense that one is a constant-factor approximation of the other.

The results of this paper were announced in the extended abstract "Dynamic Programming for Graphs on Surfaces. Proceedings of ICALP 2010, volume 6198 of LNCS, pages 372-383", which is a combination of the algorithmic framework presented in this paper and the enumerative results that can be found in [Rué et al. 2011].

The research of the first author was done at the Laboratorie d'Informatique, École Polytechnique, 91128 PalaiseauCedex, France and was supported by the European Research Council under the European Community's 7th Framework Programme, ERC grant agreement 208471 - ExploreMaps project. The research of the second author was supported by projects ANR Agape and ANR Gratos. The research of the last author was supported by Project "Kapodistrias" (AП 02839/28.07.2008) of the National and Kapodistrian University of Athens (project code: 70/4/8757). Author's addresses: Juanjo Rué, Instituto de Ciencias Matemáticas (CSIC-UAM-UC3M-UCM), 28049 Madrid, Spain; Ignasi Sau, AlGCo project-team, CNRS, Laboratoire d'Informatique, de Robotique et de Microélectronique de Montpellier (LIRMM), Montpellier, France; Dimitrios M. Thilikos, Department of Mathematics, National and Kapodistrian University of Athens, Panepistimioupolis, 157 84, Athens, Greece.

Permission to make digital or hard copies of part or all of this work for personal or classroom use is granted without fee provided that copies are not made or distributed for profit or commercial advantage and that copies show this notice on the first page or initial screen of a display along with the full citation. Copyrights for components of this work owned by others than ACM must be honored. Abstracting with credit is permitted. To copy otherwise, to republish, to post on servers, to redistribute to lists, or to use any component of this work in other works requires prior specific permission and/or a fee. Permissions may be requested from Publications Dept., ACM, Inc., 2 Penn Plaza, Suite 701, New York, NY 10121-0701 USA, fax +1 (212) 869-0481, or permissions@acm.org.

(C) - ACM 1549-6325/-/-ART- $\$ 10.00$

DOI 10.1145/0000000.0000000 http://doi.acm.org/10.1145/0000000.0000000
} 
Dorn et al. 2007]). In this paper, we provide a general framework for the design and analysis of dynamic programming algorithms for graphs embedded in surfaces where $f(\mathbf{b w})=2^{O \text { (bw) }}$.

Dynamic programming.. Dynamic programming is applied in a bottom-up fashion on a rooted branch decomposition of the input graph $G$. Roughly, a branch decomposition of a graph is a way to decompose it into a tree structure of edge bipartitions (the formal definition is in Section 2). Each bipartition defines a separator $S$ of the graph called the middle set, of cardinality bounded by the branchwidth of the input graph. The decomposition is routed in the sense that one of the parts of each bipartition is the "lower part of the middle set", i.e., the so-far processed one. For each graph problem, dynamic programming usually requires a suitable definition of tables encoding how potential (global) solutions of the problem are restricted to a middle set and the corresponding lower part. The size of these tables reflects the dependence on $k=|S|$ in the running time of the dynamic programming.

Designing the tables for each middle set $S$ is not always an easy task and may vary considerably due to the particularities of each problem. The simplest cases are problems such as VERTEX COVER and Dominating SET, where the certificate of the solution is a set of vertices whose choice is not restricted by some global condition. This directly yields the desired $2^{O(k)}$ upper bound on their size. For other problems, such as LONGEST PATH, CyCLE PACKING, or HAMILTONIAN CYCLE, things are more complicated as the tables encode pairings of vertices of $S$, of which there are $2^{\Theta(k \log k)}$ many. However, for such problems one can do better for planar graphs following the approach introduced in [Dorn et al. 2010]. The idea in [Dorn et al. 2010] is to use a special type of branch decomposition called a sphere cut decomposition that can guarantee that the pairings are non-crossing pairings around a virtual edge-avoiding cycle (called a noose) of the plane where $G$ is embedded. This restricts the number of tables corresponding to a middle set $S$ by the $k$-th Catalan number, which is single-exponential in $k$. The same approach was extended for graphs embedded in surfaces of genus $\gamma$ [Dorn et al. 2006]. The idea in [Dorn et al. 2006] was to perform a planarization of the input graph by splitting the potential solution into at most $\gamma$ pieces and then applying the sphere cut decomposition technique of [Dorn et al. 2010] to a more general version of the problem where the number of pairings is still bounded by some Catalan number (see also [Dorn et al. 2008] for the application of this technique for more general graphs).

A wider family of problems are those where the tables of dynamic programming encode connected packings of $S$ into sets, i.e., collections of subsets of $S$ that are pairwise disjoint and where each subset is a connected part of a partial solution (see Section 3 for the formal definitions). Throughout this paper, we call these problems connected packing-encodable. Typical problems of this type are CONNECTED VERTEX COVER, ConNeCTED Dominating SET, FEedback Vertex Set (FVS), or STEInER TREE, where the connected components of a potential solution can be encoded by a collection of disjoint subsets of $S$, each of arbitrary cardinality. Here, the general bound on the table size is given by the $k$-th Bell number, and thus it can again be $2^{\Theta(k \cdot \log k)}$. To exemplify the differences between distinct types of dynamic programming encodings, we accompany this paper with an Appendix where typical dynamic programming algorithms for VERTEX COVER and CONNECTED VERTEX CovER are presented (an expert reader may safely skip these examples). Unfortunately, for the latter category of problems, none of the current techniques has been able to drop the $2^{\Theta(k \cdot \log k)}$ bound to a single-exponential one for graphs embedded in surfaces. It is worth mentioning that, according to the recent lower bounds given by Lokshtanov et al. [Lokshtanov et al. 2011b], the bound $2^{\Theta(k \cdot \log k)}$ is best possible in general graphs for some parameterized problems like Disjoint PATHs, unless the Exponential Time Hypothesis (ETH) fails.

Our results. In this paper, we follow a different approach in order to design singleexponential (in bw) algorithms for graphs embedded in surfaces. In particular, we deviate significantly from the planarization technique of [Dorn et al. 2006], which is not able to tackle problems whose solutions are encoded by general packings. Instead, we extend the concept of sphere cut decomposition from planar graphs to generic surfaces, and we exploit directly the combinatorial structure of the potential solutions in the topological surface. Our approach permits us to provide in a unified way a single-exponential (in bw) asymptotic time analysis for all aforementioned problems. Examples of other such problems are MAXIMUM Leaf Spanning Tree, Maximum Full-Degree Spanning Tree, Maximum Leaf Tree, Maximum $d$-Degree-Bounded Connected Subgraph, Metric TSP, or Maximum $d$ DEGREE-BOUNDED CONNECTED INDUCED SUBGRAPH and all the variants studied in [Sau and Thilikos 2010]. Our results are formally described in Section 3 and imply all the results 
in [Dorn et al. 2006; Dorn et al. 2010], with running times whose dependence on genus is better than the ones in [Dorn et al. 2006], as discussed in Section 9.

Our techniques.. For our results we enhance the current technology of dynamic programming using, among others, tools from topological graph theory. Our goal is to define a special type of branch decomposition of embedded graphs with nice topological properties, which we call surface cut decomposition. Moreover, we prove that such decomposition can be constructed in single-exponential time. Surface cut decompositions are based on the concept of polyhedral decomposition, which can be constructed in polynomial time. In the middle sets of a surface cut decomposition, all vertices, except possibly a set of cardinality $O(\gamma)$, are situated along a set of $O(\gamma)$ nooses of the surface with $O(\gamma)$ common points. This topological property of the middle sets is the source of the single-exponentiality of the size of the tables in dynamic programming: they correspond to non-crossing packings of a set where all its vertices, except possibly a set of cardinality $O(\gamma)$, lie on the boundary of a surface. Our next step is to reduce the problem of counting such packings to the counting of non-crossing partitions of vertices on the boundary of the same surface. Then, the single-exponential bound follows by the recent enumerative results of [Rué et al. 2011].

For performing dynamic programming, our approach resides in a common preprocessing step that is to construct a surface cut decomposition. Then, what remains is just to run a problem-specific dynamic programming algorithm on such a decomposition. The exponential bound on the size of the tables of the dynamic programming algorithm follows as a result of the enumeration analysis in Section 8 . We would like to mention here that in our algorithms we assume that an embedding of the input graph is given, but we do not need a given branch decomposition (see the end of Section 3 for more details).

Very recently, a new framework for obtaining randomized single-exponential algorithms parameterized by treewidth in general graphs has appeared in [Cygan et al. 2011]. This framework is based on a dynamic programming technique named Cut\&Count, which seems applicable to most connected packing-encodable problems, like CONNECTED VERTEX COVER, Connected Dominating Set, Feedback Vertex Set, or Steiner Tree. The randomization in the algorithms of [Cygan et al. 2011] comes from the usage a probabilistic result called the Isolation Lemma [Mulmuley et al. 1987], whose derandomization is a challenging open problem [Arvind and Mukhopadhyay 2008]. Therefore, the existence of deterministic single-exponential algorithms parameterized by treewidth for connected packing-encodable problems in general graphs remains wide open. Our results for graphs on surfaces, as well as their generalization to any proper minor-free graph family [Rué et al. 2012], can be seen as an intermediate step towards an eventual positive answer to this question.

Organization of the paper. In Section 2, we give the definitions of the main topological and graph theoretical concepts and tools that we use in this paper. In Section 3, we define formally the class of connected packing-encodable problems and we formally settle the combinatorial problem of their enumeration. In Section 4, we define the concept of a polyhedral decomposition. In section 5 , we give some results on the behavior of certain width parameters on surfaces and in Section 6, we prove some graph-topological results. The concept of a surfacecut decompositions, as well as the algorithm for its construction, are given in Section 7 . The enumeration results of the paper are presented in Section 8. Finally, some conclusions and open problems are given in Section 9.

\section{PRELIMINARIES}

Graphs.. We use standard graph terminology, see for instance [Diestel 2005]. All graphs are finite and undirected. Given a graph $G$ and an edge $e \in E(G)$, let $G / e$ be the graph obtained from $G$ by contracting $e$, removing loops and parallel edges. If $H$ can be obtained from a subgraph of $G$ by a (possibly empty) sequence of edge contractions, we say that $H$ is a minor of $G$. Given a vertex $u$ with degree two, by dissolving $u$ we denote the operation of replacing $u$ and its two incident edges by an edge between its neighbors.

Topological surfaces. In this paper, surfaces are compact and their boundary is homeomorphic to a finite set (possibly empty) of disjoint circles. We denote by $\beta(\Sigma)$ the number of connected components of the boundary of a surface $\Sigma$. The Surface Classification Theorem [Mohar and Thomassen 2001, Theorem 3.1.3] asserts that a compact and connected surface without boundary is determined, up to homeomorphism, by its Euler characteristic $\chi(\Sigma)$ and by whether it is orientable or not. More precisely, orientable surfaces are obtained by adding $g \geq 0$ handles to the sphere $\mathbb{S}^{2}$, obtaining the $g$-torus $\mathbb{T}_{g}$ with Euler characteristic 
$\chi\left(\mathbb{T}_{g}\right)=2-2 g$, while non-orientable surfaces are obtained by adding $h>0$ cross-caps to the sphere, hence obtaining a non-orientable surface $\mathbb{P}_{h}$ with Euler characteristic $\chi\left(\mathbb{P}_{h}\right)=2-h$. A subset $\Pi$ of a surface $\Sigma$ is surface-separating if $\Sigma \backslash \Pi$ has at least two connected components.

As a conclusion, our surfaces are determined, up to homeomorphism, by their orientability, their Euler characteristic, and the number of connected components of their boundary. For computational simplicity, it is convenient to work with the Euler genus $\gamma(\Sigma)$ of a surface $\Sigma$, which is defined as $\gamma(\Sigma)=2-\chi(\Sigma)$.

Graphs embedded in surfaces.. Our main reference for graphs on surfaces is the monograph of Mohar and Thomassen [Mohar and Thomassen 2001]. For a graph $G$ we use the notation $(G, \tau)$ to denote that $\tau$ is an embedding of $G$ in $\Sigma$ (that is, a drawing without edge crossings), whenever the surface $\Sigma$ is clear from the context. An embedding has vertices, edges, and faces, which are zero-, one-, and two-dimensional open sets, and are denoted $V(G), E(G)$, and $F(G)$, respectively. The degree $\mathrm{d}(v)$ of a vertex $v$ is the number of edges incident with $v$, counted with multiplicity (loops are counted twice).

For a graph $G$, the Euler genus of $G$, denoted $\gamma(G)$, is the smallest Euler genus among all surfaces in which $G$ can be embedded. Determining the Euler genus of a graph is an NPhard problem [Thomassen 1989], hence we assume throughout the paper that we are given an already embedded graph. An $O$-arc is a subset of $\Sigma$ homeomorphic to $\mathbb{S}^{1}$. A subset of $\Sigma$ meeting the embedding only at vertices of $G$ is called $G$-normal. If an $O$-arc is $G$-normal, then we call it a noose. The length of a noose is the number of its vertices. Many results in topological graph theory rely on the concept of representativity [Seymour and Thomas 1994; Robertson and Seymour 1995], also called face-width, which is a parameter that quantifies local planarity and density of embeddings. The representativity $\operatorname{rep}(G, \tau)$ of a graph embedding $(G, \tau)$ is the smallest length of a non-contractible (i.e., non null-homotopic) noose in $\Sigma$. We call an embedding $(G, \tau)$ polyhedral [Mohar and Thomassen 2001, page 151] if $G$ is 3-connected and $\operatorname{rep}(G, \tau) \geq 3$, or if $G$ is a clique and $1 \leq|V(G)| \leq 3$. With abuse of notation, we also say in that case that the graph $G$ itself is polyhedral.

For a given embedding $(G, \tau)$, we denote by $\left(G^{*}, \tau^{*}\right)$ its dual embedding. Thus $G^{*}$ is the geometric dual of $G$. Each vertex $v$ (resp. face $r$ ) in $(G, \tau)$ corresponds to some face $v^{*}$ (resp. vertex $\left.r^{*}\right)$ in $\left(G^{*}, \tau^{*}\right)$. Also, given a set $S \subseteq E(G)$, we denote by $S^{*}$ the set of edges dual to the edges in $S$. Let $(G, \tau)$ be an embedding and let $\left(G^{*}, \tau^{*}\right)$ be its dual. We define the radial graph embedding $\left(R_{G}, \tau^{R}\right)$ of $(G, \tau)$ (also known as vertex-face graph embedding) as follows: $R_{G}$ is an embedded bipartite graph with vertex set $V\left(R_{G}\right)=V(G) \cup V\left(G^{*}\right)$. For each pair $e=\{v, u\}, e^{*}=\left\{u^{*}, v^{*}\right\}$ of dual edges in $G$ and $G^{*}, R_{G}$ contains edges $\left\{v, v^{*}\right\},\left\{v^{*}, u\right\},\left\{u, u^{*}\right\}$, and $\left\{u^{*}, v\right\}$. Mohar and Thomassen [Mohar and Thomassen 2001] proved that, if $|V(G)| \geq 4$, the following conditions are equivalent: $(i)(G, \tau)$ is a polyhedral embedding; (ii) $\left(G^{*}, \tau^{*}\right)$ is a polyhedral embedding; and (iii) $\left(R_{G}, \tau^{R}\right)$ has no multiple edges and every 4-cycle of $R_{G}$ is the border of some face. The medial graph embedding $\left(M_{G}, \tau^{M}\right)$ of $(G, \tau)$ is the dual embedding of the radial embedding $\left(R_{G}, \tau^{R}\right)$ of $(G, \tau)$. Note that $\left(M_{G}, \tau^{M}\right)$ is a $\Sigma$-embedded 4-regular graph.

Tree-like decompositions of graphs. Let $G$ be a graph on $n$ vertices. A branch decomposition $(T, \mu)$ of a graph $G$ consists of an unrooted ternary tree $T$ (i.e., all internal vertices are of degree three) and a bijection $\mu: L \rightarrow E(G)$ from the set $L$ of leaves of $T$ to the edge set of $G$. We define for every edge $e$ of $T$ the middle set $\operatorname{mid}(e) \subseteq V(G)$ as follows: Let $T_{1}$ and $T_{2}$ be the two connected components of $T \backslash\{e\}$. Then let $G_{i}$ be the graph induced by the edge set $\left\{\mu(f): f \in L \cap V\left(T_{i}\right)\right\}$ for $i \in\{1,2\}$. The middle set is the intersection of the vertex sets of $G_{1}$ and $G_{2}$, i.e., $\operatorname{mid}(e):=V\left(G_{1}\right) \cap V\left(G_{2}\right)$. The width of $(T, \mu)$ is the maximum order of the middle sets over all edges of $T$, i.e., $\mathbf{w}(T, \mu):=\max \{|\operatorname{mid}(e)| \mid e \in T\}$. An optimal branch decomposition of $G$ is defined by a tree $T$ and a bijection $\mu$ which give the minimum width, the branchwidth, denoted by bw $(G)$. By definition (see [Robertson and Seymour 1991]), the branchwidth of a graph $G$ with $|E(G)| \leq 1$ is taken to be zero.

Let $G=(V, E)$ be a connected graph. For $S \subseteq V$, we denote by $\delta(S)$ the set of all edges with an end in $S$ and an end in $V \backslash S$. Let $\left\{V_{1}, V_{2}\right\}$ be a partition of $V$. If $G\left[V_{1}\right]$ and $G\left[V_{2}\right]$ are both non-null and connected, we call $\delta\left(V_{1}\right)$ a bond of $G$ [Seymour and Thomas 1994].

A carving decomposition $(T, \mu)$ is similar to a branch decomposition, only with the difference that $\mu$ is a bijection between the leaves of the tree and the vertex set of the graph $G$. For an edge $e$ of $T$, the counterpart of the middle set, called the cut set cut $(e)$, contains the edges of $G$ with endvertices in the leaves of both subtrees. The counterpart of branchwidth is carvingwidth, and is denoted by $\mathrm{cw}(G)$. In a bond carving decomposition, every cut set is a bond of the graph. That is, in a bond carving decomposition, every cut set separates the graph into two connected components. 
Let $G_{1}$ and $G_{2}$ be graphs with disjoint vertex-sets and let $k \geq 0$ be an integer. For $i=1,2$, let $W_{i} \subseteq V\left(G_{i}\right)$ form a clique of size $k$ and let $G_{i}^{\prime}(i=1,2)$ be obtained from $G_{i}$ by deleting some (possibly no) edges from $G_{i}\left[W_{i}\right]$ with both endvertices in $W_{i}$. Consider a bijection $h: W_{1} \rightarrow W_{2}$. We define a clique sum $G$ of $G_{1}$ and $G_{2}$, denoted by $G=G_{1} \oplus_{k} G_{2}$, to be the graph obtained from the union of $G_{1}^{\prime}$ and $G_{2}^{\prime}$ by identifying $w$ with $h(w)$ for all $w \in W_{1}$. The integer $k$ is called the size of the clique sum. Given a set of graphs $\mathcal{G}$ and an integer $\ell \geq 0$, we define the $\ell$-clique sum closure of $\mathcal{G}$ as the set of graphs $\mathcal{G}_{\ell}$ recursively defined as follows: every graph in $\mathcal{G}$ is also in $\mathcal{G}_{\ell}$, and if $G_{1} \in \mathcal{G}, G_{2} \in \mathcal{G}_{\ell}$, and $G_{3}=G_{1} \oplus_{k} G_{2}$ with $0 \leq k \leq \ell$, then $G_{3} \in \mathcal{G}_{\ell}$.

\section{CONNECTED PACKING-ENCODABLE PROBLEMS}

The standard dynamic programming approach on branch decompositions requires the so called rooted branch decomposition, defined as a triple $\left(T, \mu, e_{r}\right)$, where $(T, \mu)$ is a branchdecomposition of $G$ such that $T$ is a tree rooted on a leaf $v_{l}$ of $T$ incident with some edge $e_{r}$. We slightly abuse notation by insisting that no edge of $G$ is assigned to $v_{l}$ and thus $\operatorname{mid}\left(e_{r}\right)=\emptyset$ (for this, we arbitrarily pick some edge of a branch decomposition, subdivide it and then connect by $e_{r}$ the subdivision vertex with a new leaf $v_{l}$ ). The edges of $T$ are oriented towards the root $e_{r}$ and for each edge $e \in E(T)$ we denote by $E_{e}$ the edges of $G$ that are mapped to leaves of $T$ that are descendants of $e$. We also set $G_{e}=G\left[E_{e}\right]$ and we denote by $L(T)$ the edges of $T$ that are incident with leaves of $T$. Given an edge $e$ whose tail is a non-leaf vertex $v$, we denote by $e_{1}, e_{2} \in E(T)$ the two edges heading at $v$ (we call them children of $e$ ). When the tail of an edge of $T$ is also a leaf of $T$ then we call it leaf-edge.

Typically, dynamic programming on a rooted branch decomposition $\left(T, \mu, e_{r}\right)$ of a graph $G$ associates some suitable combinatorial structure struct $(e)$ with each edge $e$ of $T$ such that the knowledge of struct $\left(e_{r}\right)$ makes it possible to determine the solution to the problem. Roughly speaking, struct $(e)$ encodes all the ways that the possible certificates of a partial solution on graph $G_{e}$ may be restricted to $\operatorname{mid}(e)$. The computation of struct $(e)$ is done bottom-up by first providing struct $(e)$ when $e$ is a leaf-edge of $T$ and then giving a recursive way to construct struct $(e)$ from $\operatorname{struct}\left(e_{1}\right)$ and $\operatorname{struct}\left(e_{2}\right)$, where $e_{1}$ and $e_{2}$ are the children of $e$.

The encoding of struct is commonly referred as the "tables" of the dynamic programming algorithm. It is desirable that the size of the tables, as well as the time to process them, is bounded by $f(|\operatorname{mid}(e)|) \cdot n^{O(1)}$, where $f$ is a function not depending on $n$. This would give a polynomial-time algorithm for graphs of fixed branchwidth. In technical terms, this means that the problem is Fixed Parameter Tractable (FPT), when parameterized by the branchwidth of the input graph (for more on Fixed Parameter Tractability, see [Flum and Grohe 2006; Downey and Fellows 1999; Niedermeier 2006]). A challenge in the design of such algorithms is to reduce the contribution of branchwidth to the size of their tables and therefore to simplify $f$ as much as possible. As indicated by the lower bounds in [Lokshtanov et al. 2011a; Impagliazzo et al. 2001; Cai and Juedes 2003], for many problems like INDEPENDENT SET, Dominating SET, or $q$-Coloring for fixed $q \geq 3, f$ is not expected to be better than single-exponential in general graphs.

Before we proceed with the description of the family of problems that we examine in this paper, we need some definitions. Let $G$ be a graph and let $S$ be a set of vertices of $G$. We denote by $\mathcal{G}$ the collection of all subgraphs of $G$. Each $H \in \mathcal{G}$ defines a packing (that is, a collection of pairwise disjoint non-empty subsets) $\mathcal{P}_{S}(H)$ of $S$ such that two vertices $x, y \in S$ belong to the same set of $\mathcal{P}_{S}(H)$ if $x, y$ belong to the same connected component of $H$. We say that $H_{1}, H_{2} \in \mathcal{G}$ are $S$-equivalent if $\mathcal{P}_{S}\left(H_{1}\right)=\mathcal{P}_{S}\left(H_{2}\right)$, and we denote it by $H_{1} \equiv_{S} H_{2}$. Let $\overline{\mathcal{G}}_{S}$ the collection of all subgraphs of $G$ modulo the equivalence relation $\equiv_{S}$. We define the set of all connected packings of $S$ with respect to $G$ as the collection

$$
\Psi_{G}(S)=\left\{\mathcal{P}_{S}(H) \mid H \in \overline{\mathcal{G}}_{S}\right\} .
$$

Notice that each member of $\Psi_{G}(S)$ can indeed be seen as a packing of $S$, as its sets may not necessarily meet all vertices of $S$.

In this paper we consider graph problems that can be solved by dynamic programming algorithms on branch decompositions for which the size of $\operatorname{struct}(e)$ is upper-bounded by $2^{O(|\operatorname{mid}(e)|)} \cdot\left|\Psi_{G_{e}}(\operatorname{mid}(e))\right| \cdot n^{O(1)}$. We call these problems connected packing-encodable. We stress that our definition of connected packing-encodable problem assumes the existence of an algorithm with this property, but there may exist other algorithms whose tables are much bigger. In the introduction, we gave a long list of problems that belong to this category and, in the Appendix, we make a full description of how to do dynamic programming for one of them. For these problems, dynamic programming has a single-exponential dependance on 
branchwidth if and only if $\Psi_{G_{e}}(\operatorname{mid}(e))$ contains a single-exponential number of packings, i.e., $\left|\Psi_{G_{e}}(\operatorname{mid}(e))\right|=2^{O(|\operatorname{mid}(e)|)}$.

Indeed, assume that we have the desired bound on $\Psi_{G_{e}}(\operatorname{mid}(e))$ for all edges $e$ of a branch decomposition $(T, \mu)$ of $G$. In order to recursively compute $\Psi_{G_{e}}(\operatorname{mid}(e))$ for a given edge $e \in E(T)$ from the tables of its two children $e_{1}, e_{2} \in E(T)$, we just try of possibilities of combining a connected packing corresponding to $e_{1}$ and a connected packing corresponding to $e_{2}$ (of course, the precise way to perform this merging operation will depend on each particular problem). This procedure takes time $\left|\Psi_{G_{e_{1}}}\left(\operatorname{mid}\left(e_{1}\right)\right)\right| \cdot\left|\Psi_{G_{e_{2}}}\left(\operatorname{mid}\left(e_{2}\right)\right)\right|=2^{O\left(\left|\operatorname{mid}\left(e_{1}\right)\right|+\left|\operatorname{mid}\left(e_{2}\right)\right|\right)}=$ $2^{O(\mathbf{w}(T, \mu))}$, and the size of $\Psi_{G_{e}}(\operatorname{mid}(e))$ is $2^{O(|\operatorname{mid}(e)|)}$ by assumption.

However, in general the number of different connected packings that could be created during the dynamic programming is not necessarily smaller than the number of the nonconnected ones. Therefore, it may linearly depend on the $k$-th Bell number, where $k$ is the branchwidth of the input graph. This implies that, in general, $\left|\Psi_{G_{e}}(\operatorname{mid}(e))\right|=2^{O(k \log k)}$ is the best upper bound we may achieve for connected packing-encodable problems, at least for deterministic algorithms. The purpose of this paper is to show that, for such problems, this bound can be reduced to a single-exponential one when their input graphs have bounded genus. In Section 7, we define the concept of a surface cut decomposition, which is a key tool for the main result of this paper, summarized as follows.

THEOREM 3.1. Every connected packing-encodable problem whose input graph $G$ is embedded in a surface of Euler genus $\gamma$, and has branchwidth at most $k$, can be solved by a dynamic programming algorithm on a surface cut decomposition of $G$ with tables of size $\gamma^{O(k)} \cdot k^{O(\gamma)} \cdot \gamma^{O(\gamma)} \cdot n^{O(1)}$.

In Section 7, we prove (Theorem 7.2) that, given a graph $G$ embedded in a surface of Euler genus $\gamma$, a surface cut decomposition of $G$ of width $O(\mathbf{b w}(G)+\gamma)$ can be constructed in $2^{O(\operatorname{bw}(G))} \cdot n^{3}$ steps. Therefore, we conclude the following result.

THEOREM 3.2. Every connected packing-encodable problem whose input graph $G$ is embedded in a surface of Euler genus $\gamma$, and has branchwidth at most $k$, can be solved in $\gamma^{O(k)} \cdot k^{O(\gamma)} \cdot \gamma^{O(\gamma)} \cdot n^{O(1)}$ steps.

Given a parameterized problem with parameter $k$, an algorithm that solves it in time $2^{O(k)} \cdot n^{O(1)}$ is called a single-exponential FPT-algorithm. As finding an optimal embedding of a graph of genus $\gamma$ can be solved in $f(\gamma) \cdot n$ steps [Mohar 1999] (see also [Kawarabayashi et al. 2008] for a faster algorithm that runs in $2^{\gamma^{O(1)}} n$ steps), we can restate Theorem 3.2 as follows.

COROLLARY 3.3. Every connected packing-encodable problem on graphs of fixed genus has a single-exponential FPT-algorithm, when parameterized by the branchwidth of its input.

\section{POLYHEDRAL DECOMPOSITIONS}

We introduce in this section polyhedral decompositions of graphs embedded in surfaces. Let $G$ be an embedded graph, and let $N$ be a noose in the surface. Similarly to [Cabello and Mohar 2007], we use the notation $G \nsim N$ for the graph obtained by cutting $G$ along the noose $N$ and gluing a disk on the obtained boundaries.

Definition 4.1. Given a graph $G=(V, E)$ embedded in a surface of Euler genus $\gamma$, a polyhedral decomposition of $G$ is a set of graphs $\mathcal{G}=\left\{H_{1}, \ldots, H_{\ell}\right\}$ together with a set of vertices $A \subseteq V$ such that

$-|A|=O(\gamma)$;

- $H_{i}$ is a minor of $G[V \backslash A]$, for $i=1, \ldots, \ell$;

$-H_{i}$ has a polyhedral embedding in a surface of Euler genus at most $\gamma$, for $i=1, \ldots, \ell$;

$-G[V \backslash A]$ belongs to the 2-clique sum closure of $\mathcal{G}$.

We now rephrase the definition of a polyhedral embedding, which will we useful for our purposes (see also other equivalent definitions in [Mohar and Thomassen 2001, page 151]).

OBSERVATION 4.1. Note that an embedded graph $H$ is not polyhedral if and only if there exists a noose $N$ of length at most two in the surface in which $H$ is embedded, such that either $N$ is non-contractible or $V(H) \cap N$ separates $H$. Indeed, if $H$ has representativity at most two, then there exists a non-contractible noose $N$ of length at most two. Otherwise, since $H$ is not polyhedral, $H$ has a minimal separator $S$ of size at most two. It is then easy to see that there exists a noose containing only vertices of $S$. 
Algorithm 1 provides an efficient way to construct a polyhedral decomposition, as it is stated in Proposition 4.2. In the algorithm, the addition of an edge $\{u, v\}$ represents the existence of a path in $G$ between $u$ and $v$ that is not contained in the current component.

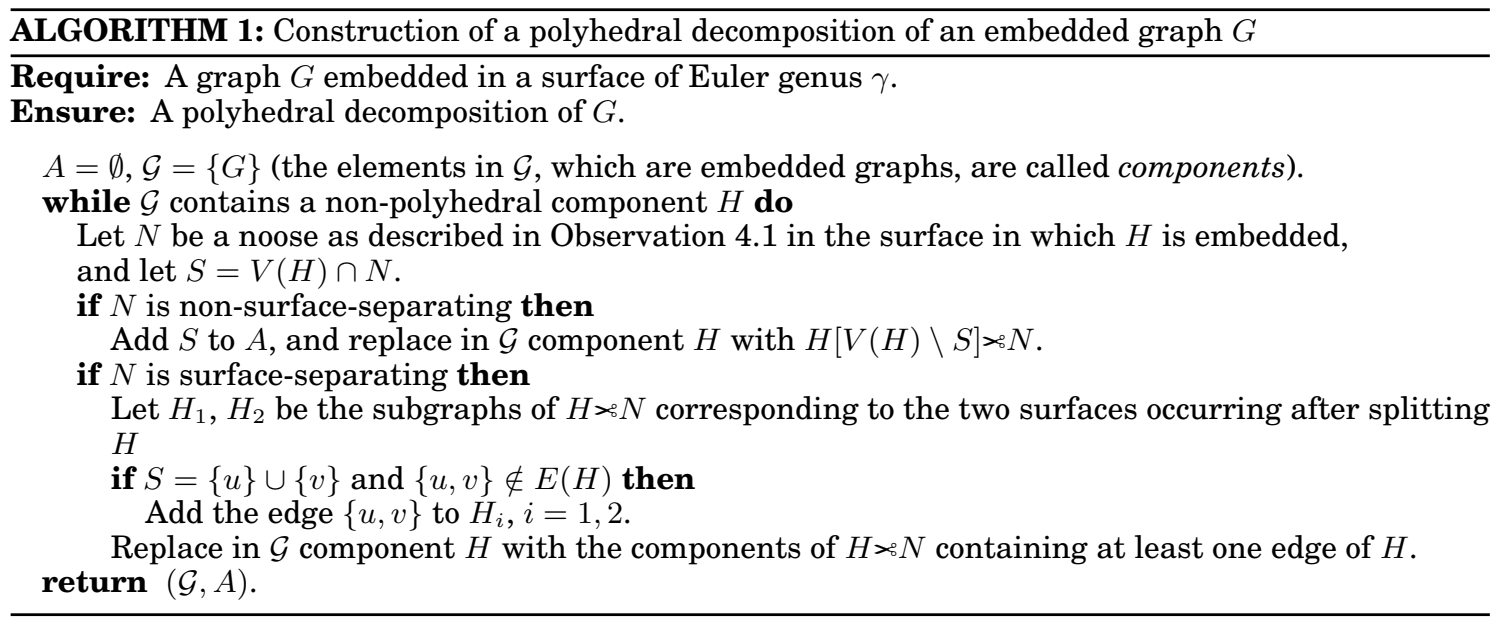

Proposition 4.2. Given a graph $G$ on $n$ vertices embedded in a surface, Algorithm 1 constructs a polyhedral decomposition of $G$ in $O\left(n^{3}\right)$ steps.

PROOF. We first prove that the the output $(\mathcal{G}, A)$ of Algorithm 1 is indeed a polyhedral decomposition of $G$, and then we analyze the running time.

Let us see that each component of $\mathcal{G}$ is a minor of $G[V \backslash A]$. Indeed, the only edges added to $G$ by Algorithm 1 are those between two non-adjacent vertices $u, v$ that separate a component $H$ into several components $H_{1}, \ldots, H_{\ell}$. For each component $H_{i}, i=1, \ldots, \ell$, there exists a path between $u$ and $v$ in $H \backslash H_{i}$ (provided that the separators of size 1 have been already removed, which can we assumed without loss of generality), and therefore the graph obtained from $H_{i}$ by adding the edge $\{u, v\}$ is a minor of $H$, which is inductively a minor of $G[V \backslash A]$. Also, each component of $\mathcal{G}$ is polyhedral by definition of the algorithm.

As a non-separating noose is necessarily non-contractible, each time some vertices are moved to $A$, the Euler genus of the surfaces strictly decreases [Mohar and Thomassen 2001, Lemma 4.2.4]. Therefore, $|A|=O(\gamma)$.

By the construction of the algorithm, it is also clear that each component of $\mathcal{G}$ has a polyhedral embedding in a surface of Euler genus at most $\gamma$. Finally, $G[V \backslash A]$ can be constructed by joining the graphs of $\mathcal{G}$ applying clique sums of size at most two.

Thus, $(\mathcal{G}, A)$ is a polyhedral decomposition of $G$ according to Definition 4.1.

We now analyze the running time of the algorithm. Separators of size at most two can be found in $O\left(n^{2}\right)$ steps [Henzinger et al. 2000]. A noose with respect to a graph $H$ corresponds to a cycle in the radial graph of $H$, hence can also be found ${ }^{2}$ in $O\left(n^{2}\right)$ (using that the number of edges of a bounded-genus graph is linearly bounded by its number of vertices). Since each time that we find a small separator we decrease the size of the components, the running time of the algorithm is $O\left(n^{3}\right)$.

\section{WIDTH PARAMETERS OF GRAPHS ON SURFACES}

In this section we state some definitions and auxiliary results about several width parameters of graphs on surfaces, to be applied in Section 7 for building surface cut decompositions. In the same spirit of [Fomin and Thilikos 2007, Theorem 1] we can prove the following lemma. We omit the proof here since the details are very similar ${ }^{3}$ to the proof in [Fomin and Thilikos 2007].

LEMMA 5.1. Let $(G, \tau)$ and $\left(G^{*}, \tau^{*}\right)$ be dual polyhedral embeddings in a surface of Euler genus $\gamma$ and let $\left(M_{G}, \tau^{M}\right)$ be the medial graph embedding. Then $\max \left\{\mathrm{bw}(G), \mathrm{bw}\left(G^{*}\right)\right\} \leq$

\footnotetext{
${ }^{2} \mathrm{~A}$ shortest non-contractible cycle can be found in $2^{O(\gamma \log \gamma)} n^{4 / 3}$ steps [Cabello and Mohar 2007]. This running time improves on $O\left(n^{3}\right)$ for a big range of values of $\gamma$.

${ }^{3}$ The improvement in the multiplicative factor of the Euler genus is obtained by applying more carefully Euler's formula in the proof analogous to that of [Fomin and Thilikos 2007, Lemma 2].
} 
$\mathbf{c w}\left(M_{G}\right) / 2 \leq 6 \cdot \mathbf{b w}(G)+2 \gamma+O(1)$. In addition, given a branch decomposition of $G$ of width at most $k$, a carving decomposition of $M_{G}$ of width at most $12 k$ can be found in linear time.

We would like to point out that in Lemma 5.1 we need the embeddings to be polyhedral.

LEMMA 5.2 (FOLKLORE). The removal of a vertex $v$ from a simple graph $G$ decreases its branchwidth by at most 1 , unless bw $(G)=2$ and $v$ is a vertex whose removal from $G$ creates a graph with all vertices of degree at most 1, in which case the branchwidth decreases by 2.

Before stating Lemma 5.5, we need two technical lemmata that will use used in the proof.

LEMMA 5.3. Let $G_{1}$ and $G_{2}$ be graphs with at most one vertex in common. Then bw $\left(G_{1} \cup\right.$ $\left.G_{2}\right)=\max \left\{\mathbf{b w}\left(G_{1}\right), \mathbf{b w}\left(G_{2}\right)\right\}$.

PRoOF. Assume first that $G_{1}$ and $G_{2}$ share one vertex $v$. Clearly bw $\left(G_{1} \cup G_{2}\right) \geq$ $\max \left\{\mathbf{b w}\left(G_{1}\right)\right.$, bw $\left.\left(G_{2}\right)\right\}$. Conversely, for $i=1,2$, let $\left(T_{i}, \mu_{i}\right)$ be a branch decomposition of $G_{i}$ such that $\mathbf{w}\left(T_{i}, \mu_{i}\right) \leq k$. For $i=1,2$, let $T_{i}^{v}$ be the minimal subtree of $T_{i}$ containing all the leaves $u_{i}$ of $T_{i}$ such that $v$ is an endvertex of $\mu_{i}\left(u_{i}\right)$. For $i=1,2$, we take an arbitrary edge $\left\{a_{i}, b_{i}\right\}$ of $T_{i}^{v}$, we subdivide it by adding a new vertex $w_{i}$, and then we build a tree $T$ from $T_{1}$ and $T_{2}$ by adding the edge $\left\{w_{1}, w_{2}\right\}$. We claim that $\left(T, \mu_{1} \cup \mu_{2}\right)$ is a branch decomposition of $G_{1} \cup G_{2}$ of width at most $k$. Indeed, let us compare the middle sets of $\left(T, \mu_{1} \cup \mu_{2}\right)$ to those of $\left(T_{1}, \mu_{1}\right)$ and $\left(T_{2}, \mu_{2}\right)$. First, it is clear that the vertices of $V\left(G_{1}\right) \cup V\left(G_{2}\right)-\{v\}$ appear in $\left(T, \mu_{1} \cup \mu_{2}\right)$ in the same middle sets as in $\left(T_{1}, \mu_{1}\right)$ and $\left(T_{2}, \mu_{2}\right)$. Secondly, mid $\left(\left\{w_{1}, w_{2}\right\}\right)=\{v\}$, since $v$ is a cut-vertex of $G_{1} \cup G_{2}$. Also, for $i=1,2, \operatorname{mid}\left(\left\{a_{i}, w_{i}\right\}\right)=\operatorname{mid}\left(\left\{w_{i}, b_{i}\right\}\right)=$ $\operatorname{mid}\left(\left\{a_{i}, b_{i}\right\}\right)$, and the latter has size at most $k$ as $\mathbf{w}\left(T_{i}, \mu_{i}\right) \leq k$. For all other edges $e$ of $T_{i}, i=1,2, \operatorname{mid}(e)$ is exactly the same in $T$ and in $T_{i}$, since if $e \in E\left(T_{i}^{v}\right)$ then $v \in \operatorname{mid}(e)$ in both $T$ and $T_{i}$, and if $e \in E\left(T_{i} \backslash T_{i}^{v}\right)$ then $v \notin \operatorname{mid}(e)$ in both $T$ and $T_{i}$.

If $G_{1}$ and $G_{2}$ share no vertices, we can merge two branch decompositions $\left(T_{1}, \mu_{1}\right)$ and $\left(T_{2}, \mu_{2}\right)$ by subdividing a pair of arbitrary edges, without increasing the width.

Lemma 5.4 (Fomin And Thilikos [Fomin And Thilikos 2006]). Let $G_{1}$ and $G_{2}$ be graphs with one edge $f$ in common. Then $\mathbf{b w}\left(G_{1} \cup G_{2}\right) \leq \max \left\{\mathbf{b w}\left(G_{1}\right), \mathbf{b w}\left(G_{2}\right), 2\right\}$. Moreover, if both endvertices of $f$ have degree at least two in at least one of the graphs, then $\mathbf{b w}\left(G_{1} \cup G_{2}\right)=\max \left\{\mathbf{b w}\left(G_{1}\right)\right.$, bw $\left.\left(G_{2}\right)\right\}$.

LEMMA 5.5. Let $G$ be a graph and let $\mathcal{G}$ be a collection of graphs such that $G$ can be constructed by joining graphs in $\mathcal{G}$ applying clique sums of size at most two. Given branch decompositions $\left.\left\{\left(T_{H}, \mu_{H}\right) \mid H \in \mathcal{G}\right)\right\}$, we can compute in linear time a branch decomposition $(T, \mu)$ of $G$ such that $\mathbf{w}(T, \mu) \leq \max \left\{2,\left\{\mathbf{w}\left(T_{H}, \mu_{H}\right) \mid H \in \mathcal{G}\right\}\right\}$. In particular, bw $(G) \leq$ $\max \{2,\{\mathbf{b w}(H) \mid H \in \mathcal{G}\}\}$.

Proof. Note that if $G_{1}$ and $G_{2}$ are graphs with no vertex (resp. a vertex, an edge) in common, then $G_{1} \cup G_{2}=G_{1} \oplus_{0} G_{2}$ (resp. $G_{1} \oplus_{1} G_{2}, G_{1} \oplus_{2} G_{2}$ ).

We have to show how to merge the branch decompositions $\left(T_{1}, \mu_{1}\right),\left(T_{2}, \mu_{2}\right)$ of two graphs $H_{1}, H_{2}$ in $\mathcal{G}$. We distinguish four cases:

(a) $H_{1}$ and $H_{2}$ share two vertices $v_{1}, v_{2}$, and the edge $e=\left\{v_{1}, v_{2}\right\} \in E(G)$. We take the leaves in $T_{1}$ and $T_{2}$ corresponding to $e$, we identify them, and we add a new edge whose leave corresponds to $e$ (see Figure 1(a)).

(b) $H_{1}$ and $H_{2}$ share two vertices $v_{1}, v_{2}$, and the edge $e=\left\{v_{1}, v_{2}\right\} \notin E(G)$. We take the leaves in $T_{1}$ and $T_{2}$ corresponding to $e$, we identify them, and we dissolve the common vertex (see Figure 1(b)).

(c) $H_{1}$ and $H_{2}$ share one vertex $v$. We take two edges $b, c$ in $T_{1}, T_{2}$ whose leaves correspond to edges containing $v$, we subdivide them and add a new edge between the newly created vertices (see Figure 1(c)).

(d) $H_{1}$ and $H_{2}$ share no vertices. We do the construction of case (c) for any two edges of the two branch decompositions.

The above construction does not increase the branchwidth by Lemmata 5.3 and 5.4 (in these two lemmata, the merging operations are exactly those described above).

Observation 5.1. Let $G=(V, E)$ be a graph, and let $A \subseteq V$. Given a branch decomposition $\left(T^{\prime}, \mu^{\prime}\right)$ of $G[V \backslash A]$, we can obtain a branch decomposition $(T, \mu)$ of $G$ with $\mathbf{w}(T, \mu) \leq \mathbf{w}\left(T^{\prime}, \mu^{\prime}\right)+|A|$ recursively as follows: First, for each edge $\{u, v\} \in E(G)$ with $u \in V \backslash A$ 


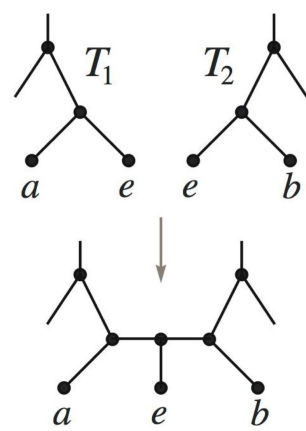

(a)

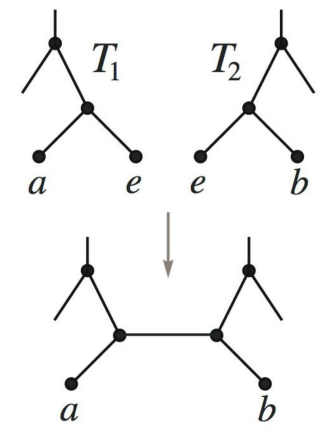

(b)

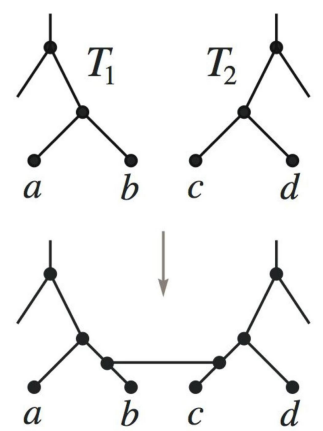

(c)

Fig. 1. Merging branch decompositions $\left(T_{1}, \mu_{1}\right)$ and $\left(T_{2}, \mu_{2}\right)$ of two components $H_{1}$ and $H_{2}$ in a polyhedral decomposition $(\mathcal{G}, A)$ of $G=(V, E)$. There are three cases: (a) $H_{1}$ and $H_{2}$ share two vertices $v_{1}, v_{2}$ and the edge $e=\left\{v_{1}, v_{2}\right\}$ is in $E$; (b) $H_{1}$ and $H_{2}$ share two vertices $v_{1}, v_{2}$ and $e=\left\{v_{1}, v_{2}\right\}$ is not in $E$; (c) $H_{1}$ and $H_{2}$ share one vertex $v$.

and $v \in A$, we choose an edge $e \in G[V \backslash A]$ containing $u$, and we replace the leaf of $T^{\prime}$ corresponding to $e$ with two incident pendant edges whose leaves correspond to edges $\{u, v\}$ and $e$, respectively. Finally, for each edge $\{u, v\} \in E(G)$ with $u, v \in A$, we take and arbitrary edge of $T^{\prime}$, subdivide it, and add a new edge whose leave corresponds to edge $\{u, v\}$. It can be easily checked that the size of the middle sets has increased by at most $|A|$.

Given an embedded graph $G$ and a carving decomposition $(T, \mu)$ of its medial graph $M_{G}$, we define a radial decomposition $\left(T^{*}, \mu^{*}\right)$ of the dual graph $R_{G}$ as follows: let $T^{*}=T$ and let $\mu^{*}$ be a bijection from the leaves of $T$ to the set of faces of $R_{G}$ defined in the following way: for each edge $e \in E(T), \mu^{*}(e)=f$, where $f$ is the face in $R_{G}$ corresponding to the vertex $u_{f} \in V\left(M_{G}\right)$ such that $\mu(e)=u_{f}$. Each edge $e \in E\left(T^{*}\right)$ partitions the faces of $R_{G}$ into two sets $F_{1}$ and $F_{2}$. We define the border set of $e$, denoted bor $(e)$, as the set of edges of $R_{G}$ that belong to both $F_{1}$ and $F_{2}$. Note that $F_{1}$ and $F_{2}$ may intersect also in vertices, not only in edges.

If $(T, \mu)$ is a bond carving decomposition of $M_{G}$, then the associated radial decomposition (also called bond) has nice connectivity properties. Indeed, in a bond carving decomposition, every cut set partitions the vertices of $M_{G}$ into two subsets $V_{1}, V_{2}$ such that both $M_{G}\left[V_{1}\right]$ and $M_{G}\left[V_{2}\right]$ are non-null and connected. This property, seen in the radial decomposition of $R_{G}$, implies that each edge $e \in E\left(T^{*}\right)$ corresponds to a partition of the faces of $R_{G}$ into two sets $F_{1}$ and $F_{2}$, namely black and white faces (naturally partitioning the edges into black, white, and grey), such that it is possible to reach any black (resp. white) face from any black (resp. white) face by only crossing black (resp. white) edges. In other words, the union of all black (resp. white) faces and edges is a connected set.

OBSERVATION 5.2. Recall that all the faces of a radial graph $R_{G}$ are tiles, that is, each face has exactly 4 edges. Also, each one of those tiles corresponds to a pair of dual edges e and $e^{*}$ of $G$ and $G^{*}$, respectively. Given a carving decomposition $(T, \mu)$ of $M_{G}$ (or equivalently, a radial decomposition $\left(T^{*}, \mu^{*}\right)$ of $R_{G}$ ), one can obtain in a natural way branch decompositions of $G$ and $G^{*}$ by redefining the bijection $\mu$ from the leaves of $T$ to the edges of $G$ (or $G^{*}$ ) that correspond to the faces of $R_{G}$.

\section{SOME TOPOLOGICAL RESULTS}

In this section we state two topological lemmata and some definitions that will be used in Section 7. Given a collection $\mathcal{S}$ of sets, we denote their union by $\cup \mathcal{S}=\bigcup_{S \in \mathcal{S}} S$.

Given a graph $G$ embedded in a surface of Euler genus $\gamma$, its dual $G^{*}$ and a spanning tree $C^{*}$ of $G^{*}$, we call $C=\left\{e \in E(G) \mid e^{*} \in E\left(C^{*}\right)\right\}$ a spanning cotree of $G$. We define a tree-cotree partition (cf. [Eppstein 2003]) of an embedded graph $G$ to be a triple $(T, C, X)$ where $T$ is a spanning tree of $G, C$ is a spanning cotree of $G, X \subseteq E(G)$, and the three sets $E(T), C$, and $X$ form a partition of $E(G)$. See Figure 2 for an example of a tree-cotree partition of the complete graph $K_{5}$ embedded in the classical square representation of the torus. Eppstein proved [Eppstein 2003, Lemma 3.1] that if $T$ and $C^{*}$ are forests such that $E(T)$ and $C$ are disjoint, we can make $T$ become part of a spanning tree $T^{\prime}$ and $C$ become part of a spanning cotree disjoint from $T^{\prime}$, extending $T$ and $C$ to a tree-cotree decomposition. We can now announce the following lemma from [Eppstein 2003, Lemma 3.2]. 


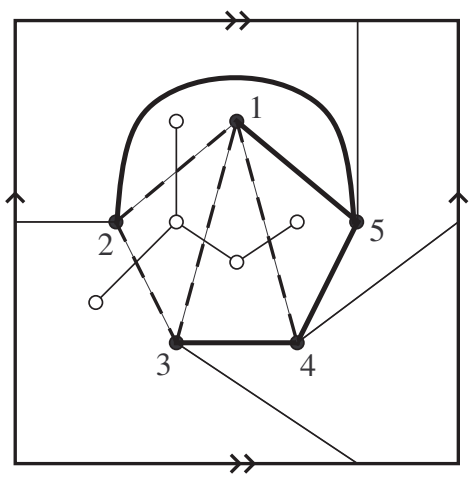

Fig. 2. Tree-cotree partition $(T, C, X)$ of the complete graph $K_{5}$ on vertices $\{1,2,3,4,5\}$ embedded in the torus. White circles correspond to the vertices of its dual $K_{5}^{*}$. For simplicity, not all edges of $K_{5}^{*}$ are drawn, only a spanning tree $C^{*}$. The corresponding spanning cotree $C$ of $K_{5}$ is drawn with dashed edges. A spanning tree $T$ of $K_{5}$ is drawn with bold edges. Finally, the set $X$ is given by the two edges $\{2,4\}$ and $\{3,5\}$.

LEMMA 6.1 (EPPSTEIN [EPPSTEIN 2003]). If $(T, C, X)$ is a tree-cotree decomposition of a graph $G$ embedded in a surface of Euler genus $\gamma$, then $|X|=O(\gamma)$.

Let $\Sigma$ be a surface and let $\mathcal{N}$ be a finite collection of $O$-arcs in $\Sigma$ pairwise intersecting at a finite zero-dimensional subsets (i.e., points) of $\Sigma$. For a point $p \in \Sigma$, let $\mathcal{N}(p)$ be the number of $O$-arcs in $\mathcal{N}$ containing $p$, and let $P(\mathcal{N})=\{p \in \Sigma: \mathcal{N}(p) \geq 2\} ;$ note that by assumption $P(\mathcal{N})$ is a finite set of points of $\Sigma$. Then we define

$$
\theta(\mathcal{N})=\sum_{p \in P(\mathcal{N})}(\mathcal{N}(p)-1) .
$$

LEMMA 6.2. Let $\Sigma$ be a surface without boundary with $\gamma(\Sigma)=\gamma$. Let $\mathcal{N}$ be a collection of $O(\gamma) O$-arcs in $\Sigma$ pairwise intersecting at finite zero-dimensional subsets of $\Sigma$, and such that $\Sigma \backslash \mathbf{U N}$ has two connected components. Then $\theta(\mathcal{N})=O(\gamma)$.

PRoof. In order to prove the lemma, we define from $\mathcal{N}$ the following (multi)graph $H$ embedded in $\Sigma$ : we first add a vertex $v_{p}$ in $H$ for every point $p$ in $\Sigma$ such that $\mathcal{N}(p) \geq 2$. We call such points repeated. We now distinguish four cases according to the number of repeated points in an $O$-arc. First, for each $O$-arc $N$ with at least three repeated points, we order cyclically the repeated points in $N$, and the same ordering applies to the corresponding vertices in $H$. Then, we add an edge in $H$ between each two consecutive vertices in that ordering. For each $O$-arc with exactly two repeated points $p$ and $q$, we add two parallel edges in $H$ between $v_{p}$ and $v_{q}$. For each $O$-arc with exactly one repeated point $p$, we add in $H$ a loop at vertex $v_{p}$. Finally, for each $O-\operatorname{arc} N$ with no repeated points, we add to $H$ a new vertex $v_{N}$ with a loop. Visually, $H$ is the graph embedded in $\Sigma$ corresponding to the union of the $O$-arcs in $\mathcal{N}$.

In order to prove the result, by the construction of $H$ it is enough to prove that $\sum_{v \in V(H)}\left(\mathrm{d}_{H}(v)-2\right)=O(\gamma)$. By assumption, $H$ separates $\Sigma$ into two connected components $\Sigma^{\prime}$ and $\Sigma^{\prime \prime}$. Let $H_{1}, H_{2}, \ldots, H_{r}$ be the maximal connected subgraphs of $H$. In particular, $r \leq|\mathcal{N}|=O(\gamma)$ by hypothesis. Some of these connected subgraphs may be incident with $\Sigma^{\prime}$ but not with $\Sigma^{\prime \prime}$, or vice-versa. Additionally, there is at least one connected subgraph $H_{i}$ incident with both connected components. Without loss of generality we assume that the subgraphs $H_{1}, H_{2}, \ldots, H_{p}$ are incident only with $\Sigma^{\prime}, H_{p+1}, \ldots, H_{q}$ are incident with both components, and $H_{q+1}, \ldots, H_{r}$ are incident only with $\Sigma^{\prime \prime}$. It is clear that there exists an $I$-arc (that is, a subset of the corresponding surface homeomorphic to the closed interval $[0,1]$ ) disjoint from all vertices and edges except in their endpoints, joining a vertex in $H_{i}$ with a vertex in $H_{i+1}$ if $1 \leq i \leq q-1$ or $p+1 \leq i \leq r-1$.

From graphs $H_{1}, H_{2}, \ldots, H_{p}, \ldots, H_{q}$ (the ones which are incident with $\Sigma^{\prime}$ ) we construct a new graph $G_{1}$ in the following inductive way: we start taking $H_{q}$ and $H_{q-1}$, and an $I$-arc joining a vertex in $H_{q}$ to a vertex in $H_{q-1}$. This $I$-arc exists because $H_{q}$ and $H_{q-1}$ are incident with $\Sigma^{\prime}$. Consider the graph obtained from $H_{q}$ and $H_{q-1}$ by adding an edge that joins this pair of vertices. Then, we delete $H_{q}$ and $H_{q-1}$ from the initial list and add this new connected 
graph. This procedure is done $q-1$ times. At the end, we obtain a connected graph $G^{\prime}$ incident with both $\Sigma^{\prime}$ and $\Sigma^{\prime \prime}$ where each vertex has degree at least three. Finally, we apply the same procedure with $G^{\prime}, H_{q+1}, \ldots, H_{r}$, obtaining a connected graph $G$. Observe also that

$$
\sum_{v \in V(H)}\left(\mathrm{d}_{H}(v)-2\right) \leq \sum_{v \in V(G)}\left(\mathrm{d}_{G}(v)-2\right)<\sum_{v \in V(G)} \mathrm{d}_{G}(v)=2|E(G)| .
$$

In what follows, we obtain upper bounds for $2|E(G)|$. Observe that $H$ defines a pair of faces over $\Sigma$, not necessarily disks. In the previous construction of $G$, every time we add an edge we either subdivide a face into two parts or not. Consequently, the number of faces that $G$ defines over $\Sigma$ is at most $2+|\mathcal{N}|$. The next step consists in reducing the surface in the following way: let $f$ be a face determined by $G$ over $\Sigma$. If $f$ is contractible, we do nothing. If it is not, there is a non-contractible $O$-arc $N$ cycle $\mathbb{S}^{1}$ contained on $f$. Let $\Sigma_{1}$ be the connected component of $\Sigma \times N$ which contains $G$. Then $G$ defines a decomposition of $\Sigma_{1}, \gamma\left(\Sigma_{1}\right) \leq \gamma$, and the number of faces has been increased by at most one. Observe that for each operation $\gg$ we reduce the Euler genus and we create at most one face. As the Euler genus is finite, so is the number of $>$ operations. This gives rise to a surface $\Sigma_{s}$ with $\gamma\left(\Sigma_{s}\right) \leq \gamma$, and such that all faces determined by $G$ are contractible. Additionally, the number of faces that $G$ determines over $\Sigma_{s}$ is smaller than $2+|\mathcal{N}|+\gamma$.

$G$ defines a map on $\Sigma_{s}$ (i.e., all faces are contractible), and consequently we can apply Euler's formula. Then $|F(G)|+|V(G)|=|E(G)|+2-\gamma\left(\Sigma_{s}\right)$. Then, as $|F(G)| \leq 2+|\mathcal{N}|+\gamma$, we obtain that $|E(G)|+2-\gamma\left(\Sigma_{s}\right)=|V(G)|+|F(G)| \leq|V(G)|+2+|\mathcal{N}|+\gamma$. The degree of each vertex is at least three, and thus $3|V(G)| \leq 2|E(G)|$. Substituting this condition in the previous equation, we obtain

$$
|E(G)|+2-\gamma\left(\Sigma_{s}\right) \leq|V(G)|+2+|\mathcal{N}|+\gamma \leq \frac{2}{3}|E(G)|+2+|\mathcal{N}|+\gamma .
$$

Isolating $|E(G)|$, we get that $2|E(G)| \leq 6|\mathcal{N}|+6 \gamma\left(\Sigma_{s}\right)+6 \gamma \leq 6|\mathcal{N}|+12 \gamma$. As by hypothesis $|\mathcal{N}|=O(\gamma)$, the previous bound yields the desired result.

\section{SURFACE CUT DECOMPOSITIONS}

Sphere cut decompositions have been introduced as a combinatorial concept in [Seymour and Thomas 1994] and were used for the first time in [Dorn et al. 2010] to analyze the running time of algorithms based on dynamic programming over branch decompositions on planar graphs (see also [Dorn et al. 2007; Sau and Thilikos 2010; Dorn et al. 2008]. In this section we generalize sphere cut decompositions to graphs on surfaces; we call them surface cut decompositions.

Definition 7.1. Given a graph $G$ embedded in a surface $\Sigma$ with $\gamma(\Sigma)=\gamma$, a surface cut decomposition of $G$ is a branch decomposition $(T, \mu)$ of $G$ such that there exists a polyhedral decomposition $(\mathcal{G}, A)$ of $G$ with the following property: for each edge $e \in E(T)$, either $\mid \operatorname{mid}(e) \backslash$ $A \mid \leq 2$, or there exists a graph $H \in \mathcal{G}$ such that

$-\operatorname{mid}(e) \backslash A \subseteq V(H)$;

— the vertices in $\operatorname{mid}(e) \backslash A$ are contained in a set $\mathcal{N}$ of nooses of $H \backslash A$ in $\Sigma$ such that

$\circ|\mathcal{N}|=O(\gamma)$

$\circ$ they pairwise intersect only at subsets of $\operatorname{mid}(e) \backslash A$;

$\circ \theta(\mathcal{N})=O(\gamma)$

$\circ \Sigma \backslash \cup \mathcal{N}$ contains exactly two connected components, such that the graph $G_{e} \backslash A$ is embedded in the closure of one of them.

Note that a sphere cut decomposition is a particular case of a surface cut decomposition when $\gamma=0$, by taking $A=\emptyset, \mathcal{G}$ containing only the graph $G$ itself, and all the vertices of each middle set contained in a single noose. We provide now an algorithm to construct a surface graph decomposition of an embedded graph. The proof of Theorem 7.2 uses Proposition 4.2 and all the results of Sections 5 and 6.

THEOREM 7.2. Given a graph $G$ on $n$ vertices embedded in a surface of Euler genus $\gamma$, with bw $(G) \leq k$, Algorithm 2 constructs, in $2^{4.5 k+O(\log k)} \cdot n^{3}$ steps, a surface cut decomposition $(T, \mu)$ of $G$ of width at most $27 k+O(\gamma)$.

PROOF. We prove, in this order, that the output $(T, \mu)$ of Algorithm 2 is indeed a surface cut decomposition of $G$, then that the width of $(T, \mu)$ is at most $27 \mathrm{bw}(G)+O(\gamma)$, and finally the claimed running time. 


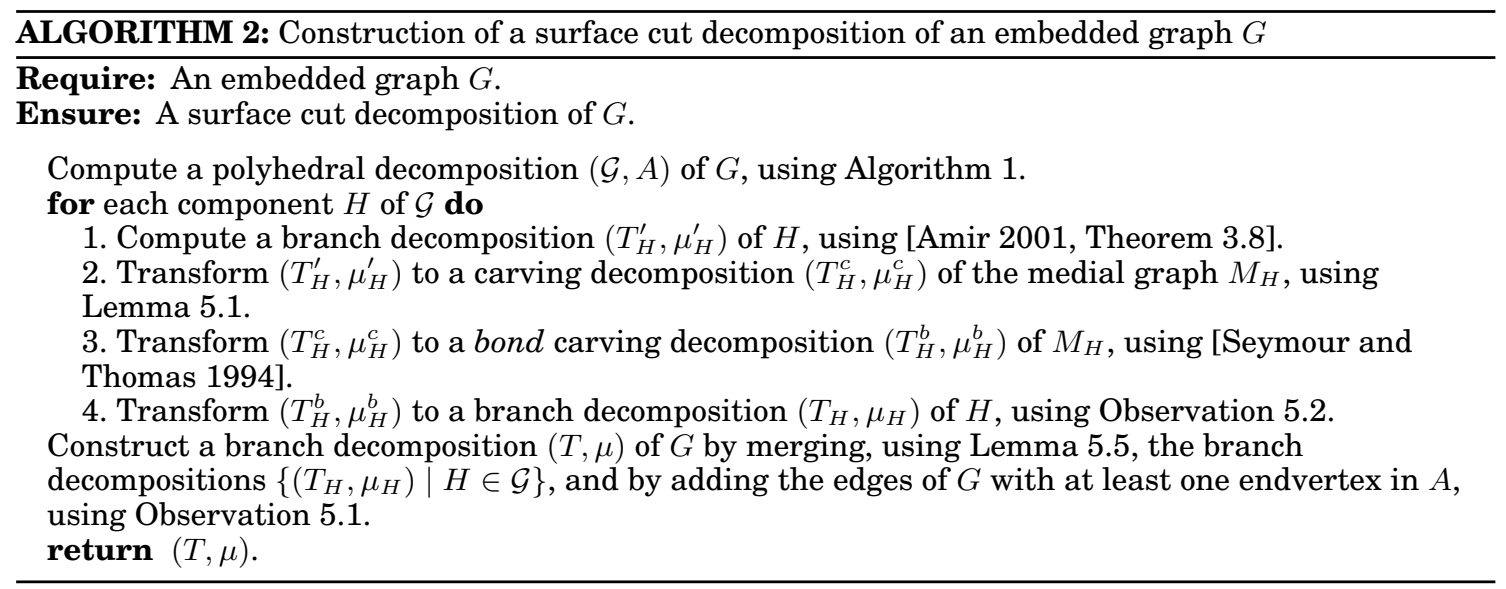

$(T, \mu)$ is a surface cut decomposition of $G$.. We shall prove that all the properties of Definition 7.1 are fulfilled. First note that, as $(\mathcal{G}, A)$ is a polyhedral decomposition of $G$, we have that $|A|=O(\gamma)$.

By construction, it is clear that $(T, \mu)$ is a branch decomposition of $G$. In $(T, \mu)$, there are some edges that have been added in the last step of Algorithm 2, in order to merge branch decompositions of the graphs in $\mathcal{G}$, with the help of Lemma 5.5. Let $e$ be such an edge. Since $(\mathcal{G}, A)$ is a polyhedral decomposition of $G$, any two graphs in $\mathcal{G}$ share at most two vertices, hence $|\operatorname{mid}(e) \backslash A| \leq 2$.

All other edges of $(T, \mu)$ correspond to an edge of a branch decomposition of some polyhedral component $H \in \mathcal{G}$. Let henceforth $e$ be such an edge. Therefore, mid $(e) \backslash A \subseteq V(H)$. To complete this part of the proof, we prove in a sequence of three claims that the remaining conditions of Definition 7.1 hold.

\section{ClaIM 7.1. The vertices in $\operatorname{mid}(e) \backslash A$ are contained in a set $\mathcal{N}$ of $O(\gamma)$ nooses.}

PROOF. The proof uses the tree-cotree partition defined in Section 6.

Recall that $e$ is an edge that corresponds to a branch decomposition $\left(T_{H}, \mu_{H}\right)$ of a polyhedral component $H$ of $\mathcal{G}$. The branch decomposition $\left(T_{H}, \mu_{H}\right)$ of $H$ has been built by Algorithm 2 from a bond carving decomposition of its medial graph $M_{H}$, or equivalently from a bond radial decomposition of its radial graph $R_{H}$. Due to the fact that the carving decomposition of $M_{H}$ is bond, edge $e$ partitions the vertices of $M_{H}$ into two sets - namely, black and white vertices each one inducing a connected subgraph of $M_{H}$. There are three types of edges in $R_{H}:$ black, white, and grey, according to whether they belong to faces of the same color (black or white) or not. Therefore, the corresponding black and white faces also induce connected subgraphs of $R_{H}$, in the sense that it is always possible to reach any black (resp. white) face from any black (resp. white) face only crossing black (resp. white) edges.

Let $F$ be the set of grey edges of $R_{H}$. Since each edge of $R_{H}$ contains a vertex from $H$ and another from $H^{*}$, the vertices in $\operatorname{mid}(e)$ are contained in $R_{H}[F]$, so it suffices to prove that $R_{H}[F]$ can be partitioned into a set of $O(\gamma)$ cycles (possibly sharing some vertices). Note that each cycle in the radial graph $R_{H}$ corresponds to a noose in the surface.

To this end, first note that in $R_{H}[F]$ all vertices have even degree. Indeed, let $v \in V\left(R_{H}[F]\right)$, and consider a clockwise orientation of the edges incident with $v$ in $R_{H}[F]$. Each such edge alternates from a black to a white face, or viceversa, so beginning from an arbitrary edge and visiting all others edges in the clockwise order, we deduce that the number of edges incident with $v$ is necessarily even.

Therefore, $R_{H}[F]$ can be partitioned into a set of cycles. Let us now bound the number of such cycles. Since the subgraph induced by the black (resp. white) faces of $R_{H}$ is connected, we can consider in $M_{H}$ a spanning tree $T_{B}^{*}$ (resp. $T_{W}^{*}$ ) corresponding to the black (resp. white) faces of $R_{H}$. Merge both trees by adding a new edge $e_{0}^{*}$, and let $T^{*}$ be the resulting tree. Let $T$ be a spanning tree of $R_{H}$ disjoint from $T^{*}$ (in the sense that there is no pair of dual edges $e$ and $e^{*}$ with $e \in E(T)$ and $e^{*} \in E\left(T^{*}\right)$ ); such a spanning tree exists by [Eppstein 2003, Lemma 3.1]. Now consider the tree-cotree partition $\left(T, T^{*}, X\right)$, where $X$ is the set of edges of $R_{H}$ that are neither in $T$ nor in $T^{*}$.

Each edge of $T^{*}$, except $e_{0}^{*}$, corresponds to two faces of $R_{H}$ of the same color. Therefore, the set $F \in E\left(R_{H}\right)$ of edges separating faces of different color is contained in $T \cup\left\{e_{0}\right\} \cup X$. Since 
$T$ is a tree, each cycle of $R_{H}[F]$ uses at least one edge in $\left\{e_{0}\right\} \cup X$. Therefore, $R_{H}[F]$ can be partitioned into at most $1+|X|$ cycles. The result follows from the fact that $\left(T, T^{*}, X\right)$ is a tree-cotree partition, and therefore $|X|=O(\gamma)$ by Lemma 6.1.

Claim 7.2. Let $\mathcal{N}$ be the set of nooses constructed in the proof of Claim 7.1. Then $\cup \mathcal{N}$ separates $\Sigma$ into two connected components.

PROof. By Claim 7.1, the vertices in $\operatorname{mid}(e) \backslash A$ are contained in $\cup \mathcal{N}$. The claim holds from the fact that for each component $H$ of $\mathcal{G},\left(T_{H}^{b}, \mu_{H}^{b}\right)$ is a bond carving decomposition of $M_{H}$, and by taking into account the discussion before Observation 5.2.

Note that the collection of nooses constructed in the proof of Claim 7.1 is finite and its elements pairwise intersect only at subsets of $\operatorname{mid}(e) \backslash A$, as required. In particular, for this collection $\mathcal{N}$ of nooses, the parameter $\theta(\mathcal{N})$ is well-defined (see Section 6).

CLAIM 7.3. Let $\mathcal{N}$ be the set of nooses constructed in the proof of Claim 7.1. Then $\theta(\mathcal{N})=$ $O(\gamma)$.

Proof. By Claim 7.2, UN separates $\Sigma$ into two connected components. The claim then holds by Lemma 6.2 .

The width of $(T, \mu)$ is at most $27 \cdot \mathrm{bw}(G)+O(\gamma)$.. For simplicity, let $k=\mathrm{bw}(G)$. By Proposition 4.2, each polyhedral component $H$ is a minor of $G$, hence bw $(H) \leq k$ for all $H \in \mathcal{G}$. In Step 1 of Algorithm 2, we compute a branch decomposition $\left(T_{H}^{\prime}, \mu_{H}^{\prime}\right)$ of $H$ of width at most $k^{\prime}=\frac{9}{2} k+1$, using Amir's algorithm [Amir 2001, Theorem 3.8] and the fact that for any graph $G$ with $|E(G)| \geq 3$, bw $(G) \leq \mathbf{t w}(G)+1 \leq \frac{3}{2} \mathbf{b w}(G)$ [Robertson and Seymour 1991]. In Step 2, we transform $\left(T_{H}^{\prime}, \mu_{H}^{\prime}\right)$ to a carving decomposition $\left(T_{H}^{c}, \mu_{H}^{c}\right)$ of the medial graph $M_{H}$ of $H$ of width at most $12 k^{\prime}$, using Lemma 5.1. In Step 3, we transform $\left(T_{H}^{c}, \mu_{H}^{c}\right)$ to a bond carving decomposition $\left(T_{H}^{b}, \mu_{H}^{b}\right)$ of $M_{H}$ of width at most $12 k^{\prime}$, using the algorithm of [Seymour and Thomas 1994]. Then, using Observation 5.2, we transform in Step $4\left(T_{H}^{b}, \mu_{H}^{b}\right)$ to a branch decomposition $\left(T_{H}, \mu_{H}\right)$ of $H$. By the proof of Claim 7.1, the discrepancy between $\mathbf{w}\left(T_{H}, \mu_{H}\right)$ and $\mathbf{w}\left(T_{H}^{b}, \mu_{H}^{b}\right) / 2$ is at most the bound provided by Lemma 6.2, i.e., $O(\gamma)$. Therefore, $\mathbf{w}\left(T_{H}, \mu_{H}\right) \leq 6 k^{\prime}+O(\gamma)=27 k+O(\gamma)$, for all $H \in \mathcal{G}$.

Then, we merge the branch decompositions of the polyhedral components, using Lemma 5.5, and finally we add the edges of $G$ with at least one endvertex in $A$, using Observation 5.1, to obtain a branch decomposition $(T, \mu)$ of $G$.

Combining the discussion above with Lemmata 5.2 and 5.5 and Observation 5.1, and using that $|A|=O(\gamma)$, we get that

$$
\begin{aligned}
\mathbf{w}(T, \mu) & \leq \max \left\{2,\left\{\mathbf{w}\left(T_{H}, \mu_{H}\right) \mid H \in \mathcal{G}\right\}\right\}+|A| \\
& \leq 27 k+O(\gamma)+|A| \\
& =27 k+O(\gamma) .
\end{aligned}
$$

Algorithm 2 runs in $2^{4.5 k+O(\log k)} \cdot n^{3}$ time. . We analyze sequentially the running time of each step. First, we compute a polyhedral decomposition of $G$ using Algorithm 1 in $O\left(n^{3}\right)$ steps, by Proposition 4.2. Then, we run Amir's algorithm in each component in Step 1, which takes $O\left(2^{4.5 k}(1.5 k)^{3 / 2} n^{2}\right)$ time [Amir 2001, Theorem 3.8], where we have used again that $\operatorname{tw}(G)+1 \leq$ $\frac{3}{2}$ bw $(G)$ [Robertson and Seymour 1991]. We would like to stress that this step is the only nonpolynomial procedure in the construction of surface cut decompositions. Step 2 can be done in linear time by Lemma 5.1. Step 3 can be done in $O\left(n^{2}\right)$ time [Seymour and Thomas 1994]. Step 4 takes linear time by Observation 5.2. Merging the branch decompositions can clearly be done in linear time. Finally, since any two elements in $\mathcal{G}$ share at most two vertices, the overall running time is the claimed one.

\section{UPPER-BOUNDING THE SIZE OF THE TABLES}

In this section we show that by using surface cut decompositions in order to solve connected packing-encodable problems in surface-embedded graphs, one can guarantee singleexponential upper bounds on the size of the tables of dynamic programming algorithms. Then Theorem 3.2 follows directly by the definition of a connected packing-encodable problem and the following lemma.

LEMMA 8.1. Let $G$ be a graph embedded in a surface $\Sigma$ without boundary and Euler genus $\gamma$, and let $(T, \mu)$ be a surface cut decomposition of $G$ of width at most $k$. Then for every $e \in E(T)$, $\left|\Psi_{G_{e}}(\operatorname{mid}(e))\right|=\gamma^{O(\gamma)} \cdot k^{O(\gamma)} \cdot \gamma^{O(k)}$. 
Before we give the proof of the above lemma, we first need to define formally the notion of non-crossing partitions on surfaces with boundary and then to prove some lemmata that combine elements from topology and combinatorics.

A non-crossing partition of a set of size $k$, from a combinatorial point of view, is a partition of the set $\{1,2, \ldots, k\}$ with the following property: if $\{a, b, c, d\} \subseteq\{1,2, \ldots, k\}$ with $1 \leq a<b<$ $c<d \leq k$ and some subset in the partition contains $a$ and $c$, then no other subset contains both $b$ and $d$. One can represent such a partition on a disk by placing $k$ points on the boundary of the disk, labeled consecutively, and drawing each subset as a convex polygon (also called block) on the points belonging to the subset. Then, the "non-crossing" condition is equivalent to the fact that the blocks are pairwise disjoint. See Figure 3 for some examples.
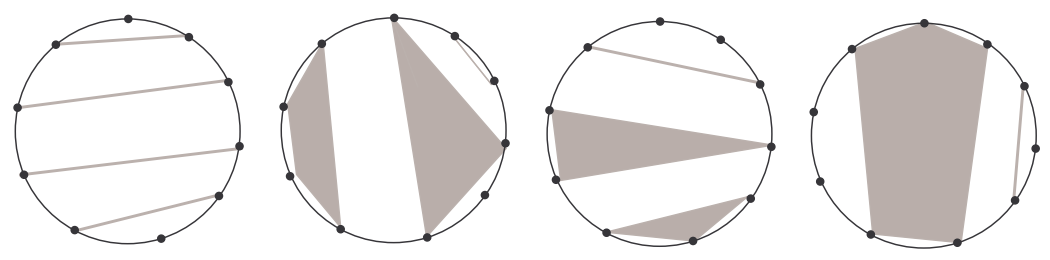

Fig. 3. Non-crossing partitions on a disk, which enumerate the number of partial solutions on planar graphs when using sphere cut decompositions.

The enumeration of non-crossing partitions on a disk is one of the first non-trivial problems in enumerative combinatorics: it is well-known (see e.g. [Flajolet and Sedgewick 2008]) that the the number of non-crossing partitions of $\{1,2, \ldots, k\}$ on a disk is equal to the Catalan number $C(k)=\frac{1}{k+1}\left(\begin{array}{c}2 k \\ k\end{array}\right) \sim \frac{4^{k}}{k^{3 / 2} \sqrt{\pi}}=O\left(4^{k}\right)$. This is the main combinatorial property exploited to obtain single-exponential dynamic programming algorithms on planar graphs using sphere cut decompositions [Dorn et al. 2010; Seymour and Thomas 1994; Sau and Thilikos 2010].

The generalization of the notion of non-crossing partition to surfaces of higher genus is not as straignforward as in the case of the disk, and must be defined carefully. We consider pairs $(\Sigma, S)$ where $\Sigma$ is a surface whose boundary has $\beta(\Sigma)$ connected components, each one homeomorphic to a simple circle, and $S$ is a set of vertices on this boundary. A partition family for the pair $(\Sigma, S)$ is a collection $\mathfrak{B}$ of mutually non-intersecting connected subsets of $\Sigma$, such that each vertex in $S$ belongs to some set in $\mathfrak{B}$.

Actually the concept of a partition family is not enough for our purposes, as we have to incorporate the presence of the set of vertices $A$ arising from a polyhedral decomposition. This set of vertices plays is some sense the role of apices in Graph Minors theory (see for instance [Robertson and Seymour 1999]), and this is why we also call these vertices apices. For this reason we consider pairs of the form $\left(\Sigma \cup \Gamma_{A}, S \cup A\right)$ where $\Sigma$ is a surface with boundary, $S$ is a set of vertices on this boundary, $A$ is a vertex set not on the boundary (the apices), and $\Gamma_{A}$ is the closed set containing the points of the graph $C_{A}$ obtained if we take a complete graph with $A$ as vertex set and add to it $S$ together with all edges between the vertices of $A$ and $S$. We require that $\Gamma_{A} \cap \Sigma=S$ and we see the set $\Gamma_{A}$ as "flying above" the surface $\Sigma$. That way, we call the edges of $C_{A}$ flying edges, and we treat them as closed subsets of $\Gamma_{A}$ by adding to them the two endpoints of their boundary. We use the notation $\Sigma^{A}$ to denote $\Sigma \cup \Gamma_{A}$ (clearly $\Sigma=\Sigma^{\emptyset}$ ). To extend the definition of partition family, we take a partition family $\mathfrak{B}_{\Sigma}$ of $\Sigma$ and, on top of it, we consider a set $\mathfrak{E}_{A}$ of flying edges where each apex is incident with some edge in $\mathfrak{E}_{A}$. An extended partition family for $\left(\Sigma^{A}, S\right)$ is a collection $\mathfrak{B}_{\Sigma^{A}}$ of subsets of $\Sigma^{A}$ defined as

$$
\mathfrak{B}_{\Sigma^{A}}=\left\{C \mid C \text { is a connected component of the } \operatorname{set} \cup\left(\mathfrak{B}_{\Sigma} \cup \mathfrak{E}_{A}\right)\right\}
$$

where $\mathfrak{B}_{\Sigma}$ and $\mathfrak{E}_{A}$ are taken as before. See Figure 4 for an example. For simplicity, we may drop the index of a collection $\mathfrak{B}_{\Sigma}$ or $\mathfrak{B}_{\Sigma^{A}}$ when it is clear from the context whether it refers to $\Sigma$ or to $\Sigma^{A}$.

Notice that each partition family $\mathfrak{B}$ for $\left(\Sigma^{A}, S \cup A\right)$ defines a partition of $S \cup A$ as follows.

$$
\mathcal{R}(\mathfrak{B})=\{(S \cup A) \cap B \mid B \in \mathfrak{B}\} .
$$

We say that two extended partition families $\mathfrak{B}_{1}$ and $\mathfrak{B}_{2}$ for $\left(\Sigma^{A}, S \cup A\right)$ are equivalent if $\mathcal{R}\left(\mathfrak{B}_{1}\right)=\mathcal{R}\left(\mathfrak{B}_{2}\right)$ and we denote it by $\mathfrak{B}_{1} \equiv \mathfrak{B}_{2}$. The set of the non-crossing partitions with apices of the set $S \cup A$ (where $S$ and $A$ are vertices embedded in $\Sigma^{A}$ as before), denoted by 


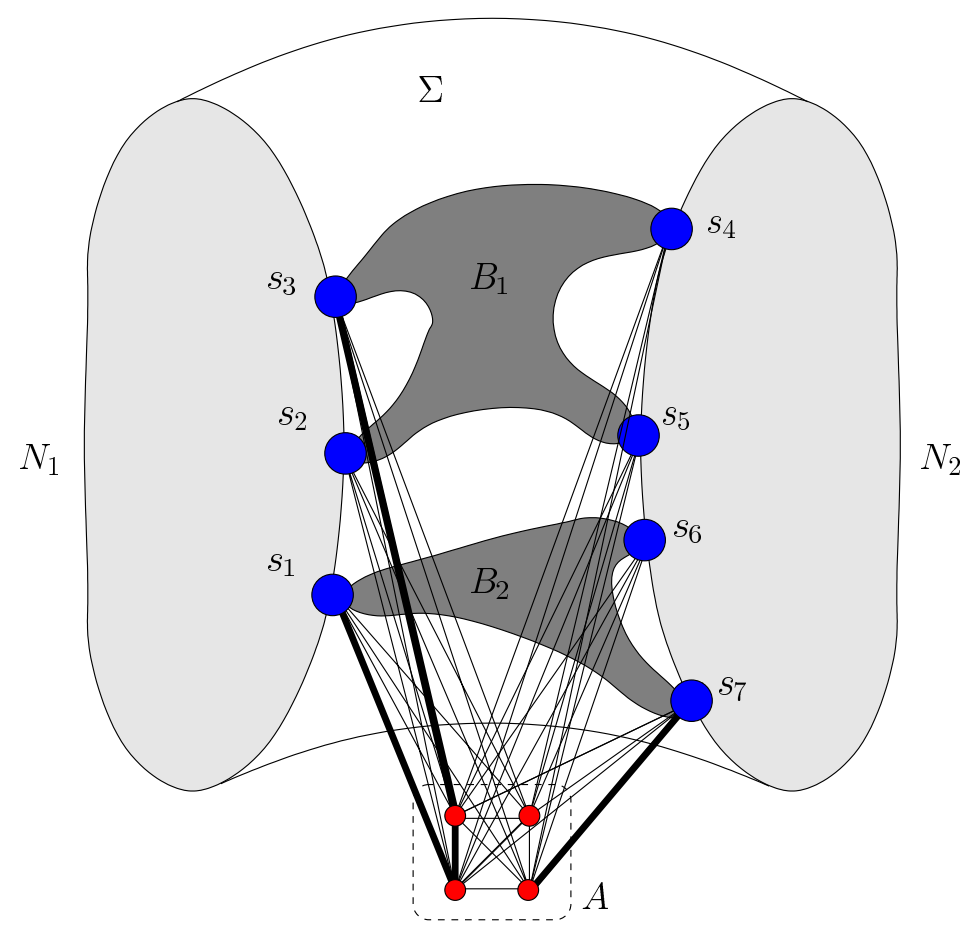

Fig. 4. An example of an extended partition family. The surface $\Sigma$ is the closure of one of the two connected components obtained by a torus after cutting along two disjoint nooses $N_{1}$ and $N_{2}$. Given that $S=\left\{s_{1}, \ldots, s_{7}\right\}$, it holds that $\mathfrak{B}_{\Sigma}=\left\{B_{1}, B_{2}\right\}$ where $B_{1}=\left\{s_{1}, s_{7}, s_{6}\right\}$ and $B_{2}=\left\{s_{2}, s_{5}, s_{4}, s_{3}\right\}$. The set $\mathfrak{C}_{A}$ contains the four bold edges. Notice that, in this case, $\mathfrak{B}_{\Sigma} A$ contains only one set consisting of the union of all the elements of $\mathfrak{B}_{\Sigma}$ and $\mathfrak{C}_{A}$.

$\Pi_{\Sigma^{A}}(S \cup A)$, is the set of equivalence classes of the extended partition families for $\left(\Sigma^{A}, S \cup A\right)$ with respect to the relation $\equiv$.

We define $\Pi_{\Sigma}(S)=\Pi_{\Sigma^{\emptyset}}(S \cup \emptyset)$, and note that, if $\Sigma$ is the disk and $|S|=k$, then $\left|\Pi_{\Sigma}(S)\right|$ is the $k$-th Catalan number and therefore $\left|\Pi_{\Sigma}(S)\right|=O\left(4^{k}\right)$. The asymptotic enumeration of $\left|\Pi_{\Sigma}(S)\right|$ for general surfaces is quite a complicated problem. However, its behavior for surfaces $\Sigma$ where $\gamma(\Sigma)$ and $\beta(\Sigma)$ are bounded is not significantly different from the disk in what concerns its exponential growth. In particular it holds that $\lim _{|S| \rightarrow \infty}\left|\Pi_{\Sigma}(S)\right|^{1 /|S|}=4$ and this is a consequence of the following enumerative result from [Rué et al. 2011].

THEOREM 8.2. Let $\Sigma$ be a surface with boundary. Then the number $\left|\Pi_{\Sigma}(S)\right|$, for $|S|=k$, verifies

$$
\left|\Pi_{\Sigma}(S)\right| \leq_{k \rightarrow \infty} \frac{C(\Sigma)}{\Gamma(3 / 2 \gamma(\Sigma)+\beta(\Sigma)-3)} \cdot k^{3 / 2 \gamma(\Sigma)+\beta(\Sigma)-4} \cdot 4^{k},
$$

where $C(\Sigma)$ is a function depending only on $\Sigma$ that is bounded by $\gamma(\Sigma)^{O(\gamma(\Sigma))}$, and $\Gamma$ is the Gamma function: $\Gamma(u)=\int_{0}^{\infty} t^{u-1} e^{-t} d t$.

The above result, which is critical for our analysis, was established using tools from analytic combinatorics (see [Flajolet and Sedgewick 2008]): singularity analysis over expressions obtained by the symbolic method. Actually, we prefer to translate it to the following looser form that is more convenient for our algorithmic purposes.

COROLlaRY 8.3. Let $\Sigma$ be a surface with boundary and let $S$ be a set of $k$ vertices in the boundary of $\Sigma$. Let also $\gamma$ be an integer such that $\gamma(\Sigma), \beta(\Sigma) \leq \gamma$. Then $\left|\Pi_{\Sigma}(S)\right| \leq \gamma^{O(\gamma)} \cdot k^{O(\gamma)} \cdot 4^{k}$.

For every set $S$ we define $\mathcal{B}(S)$ as the collection of all its partitions. Recall that if $|S|=l$, then $|\mathcal{B}(S)|$ is the $l$-th Bell number and that $|\mathcal{B}(S)|=2^{O(l \log l)}$. Also, given a collection $\mathcal{C}=$ $\left\{S_{1}, \ldots, S_{q}\right\}$ of subsets of $S$ and a subset $S^{\prime} \subseteq S$, we denote by $\left.\mathcal{C}\right|_{S^{\prime}}$ the collection of all nonempty sets in $\left\{S_{1} \cap S^{\prime}, \ldots, S_{q} \cap S^{\prime}\right\}$. Clearly, if $\mathcal{C}$ is a partition of $S$, then $\left.\mathcal{C}\right|_{S^{\prime}}$ is a partition of $S^{\prime}$.

LEMMA 8.4. Let $\Sigma$ be a surface with boundary, let $S$ be a set of vertices in the boundary of $\Sigma$, and let $A$ be a set of apices. Let also $\gamma$ and $k$ be integers such that $|A|, \gamma(\Sigma), \beta(\Sigma) \leq \gamma$ and $|S| \leq k$. Then $\left|\Pi_{\Sigma^{A}}(S \cup A)\right| \leq \gamma^{O(\gamma)} \cdot k^{O(\gamma)} \cdot \gamma^{O(k)}$. 
Proof. Let $\mathcal{R} \in \Pi_{\Sigma^{A}}(S \cup A)$ and let $\mathfrak{B}$ be an extended partition family for $\left(\Sigma^{A}, S \cup A\right)$, where $\mathcal{R}(\mathfrak{B})=\mathcal{R}$. Recall that $\mathfrak{B}_{\Sigma}$ is the set of connected components of the set $(\cup \mathfrak{B}) \cap \Sigma$. Notice that $\mathfrak{B}_{\Sigma}$ is a partition family for $(\Sigma, S)$ and thus $\mathcal{R}_{\Sigma}=\mathcal{R}\left(\mathfrak{B}_{\Sigma}\right) \in \Pi_{\Sigma}(S)$. Notice also that $\left.\mathcal{R}\right|_{A}$ is a member of $\mathcal{B}(A)$. We conclude that each $\mathcal{R} \in \Pi_{\Sigma^{A}}(S \cup A)$ uniquely generates a pair $\left(\mathcal{R}_{\Sigma},\left.\mathcal{R}\right|_{A}\right) \in \Pi_{\Sigma}(S) \times \mathcal{B}(A)$.

We define $\mathbf{P}_{\left(\mathcal{R}_{\Sigma},\left.\mathcal{R}\right|_{A}\right)}$ as the set of all possible $\mathcal{R}^{\prime}$ 's in $\Pi_{\Sigma^{A}}(S \cup A)$ that can generate a given pair $\left(\mathcal{R}_{\Sigma},\left.\mathcal{R}\right|_{A}\right) \in \Pi_{\Sigma}(S) \times \mathcal{B}(A)$.

CLAim 8.1. $\left|\mathbf{P}_{\left(\mathcal{R}_{\Sigma},\left.\mathcal{R}\right|_{A}\right)}\right| \leq\left(|\mathcal{R}|_{A} \mid+1\right)^{\left|\mathcal{R}_{\Sigma}\right|}$.

Proof. We use the notation $\left.\mathcal{R}\right|_{A}=\left\{A_{1}, \ldots, A_{q}\right\}$. Let $\mathcal{R} \in \mathbf{P}_{\left(\mathcal{R}_{\Sigma},\left.\mathcal{R}\right|_{A}\right)}$. By the above definitions, for each $i \in\{1, \ldots, q\}$, there is a unique set, say $P^{(i)}$, of $\mathcal{R}$ containing $A_{i}$ as a subset. Moreover, there is a (possibly empty) subset, say $\mathcal{B}^{(i)}$, of $\mathcal{R}_{\Sigma}$ such that $P^{(i)} \backslash A_{i}=\cup \mathcal{B}^{(i)}$. Notice that $\left\{\mathcal{B}^{(1)}, \ldots, \mathcal{B}^{(i)}\right\}$ is a packing of $\mathcal{R}_{\Sigma}$ (not necessarily a partition of $\mathcal{R}_{\Sigma}$, as some sets of $\mathcal{R}_{\Sigma}$ may appear directly as sets in $\mathcal{R}$ ). This means that each $\mathcal{R} \in \mathbf{P}_{\left(\mathcal{R}_{\Sigma},\left.\mathcal{R}\right|_{A}\right)}$ corresponds to some packing of $\mathcal{R}_{\Sigma}$ and some bijection of its sets to some of the elements of $\left.\mathcal{R}\right|_{A}$. This corresponds to the partial functions from the set $\mathcal{R}_{\Sigma}$ to the set $\left.\mathcal{R}\right|_{A}$, that is the claimed upper bound.

The rest of the proof is based on the fact that

$$
\left|\Pi_{\Sigma^{A}}(S \cup A)\right| \leq \sum_{\substack{\left(\mathcal{R}_{\Sigma},\left.\mathcal{R}\right|_{A}\right) \in \\ \Pi_{\Sigma}(S) \times \mathcal{B}(A)}}\left|\mathbf{P}_{\left(\mathcal{R}_{\Sigma},\left.\mathcal{R}\right|_{A}\right)}\right| .
$$

Recall now that $|\mathcal{B}(A)| \leq|A|^{|A|} \leq \gamma^{\gamma}$. Also, from Corollary 8.3, it holds that $\left|\Pi_{\Sigma}(S)\right|=\gamma^{O(\gamma)}$. $k^{O(\gamma)} \cdot 4^{k}$. Claim 8.1 implies that $\mathbf{P}_{\left(\mathcal{R}_{\Sigma},\left.\mathcal{R}\right|_{A}\right)} \leq(\gamma+1)^{k}$, as every packing in $\Pi_{\Sigma}(S)$ has at most $|S| \leq k$ sets and every packing in $\mathcal{B}(A)$ has at most $|A| \leq \gamma$ sets. The proof of the lemma is completed by putting all these facts together.

Let $G$ be a graph and let $S$ be a subset of $V(G)$. We define $\Pi_{G}(S)$ as the set of all partitions in $\Psi_{G}(S)$, formally,

$$
\Pi_{G}(S)=\left\{\mathcal{R} \mid \mathcal{R} \in \Psi_{G}(S) \text { and } \cup \mathcal{R}=S\right\} .
$$

LEMMA 8.5. Let $G$ be a graph and let $S^{\prime} \subseteq S \subseteq V(G)$. Then $\left|\Pi_{G}\left(S^{\prime}\right)\right| \leq\left|\Pi_{G}(S)\right|$.

PROOF. In order to prove the lemma, let us define an injective application $i: \Pi_{G}\left(S^{\prime}\right) \hookrightarrow$ $\Pi_{G}(S)$. Let $\mathcal{R} \in \Pi_{G}\left(S^{\prime}\right)$, which implies by definition (see Section 3 ) that there exists a subgraph $H \subseteq G$ whose connected components define the packing $\mathcal{R}$ of $S^{\prime}$. We define $i(\mathcal{R})$ as the packing of $S$ given by the same subgraph $H$. It is then easy to check that if $\mathcal{R}_{1}, \mathcal{R}_{2} \in \Pi_{G}\left(S^{\prime}\right)$ with $\mathcal{R}_{1} \neq \mathcal{R}_{2}$, then $i\left(\mathcal{R}_{1}\right) \neq i\left(\mathcal{R}_{2}\right)$.

LEMMA 8.6. Let $G^{\prime}$ be a graph with a set $S^{\prime} \subseteq V\left(G^{\prime}\right)$ and an edge $e=\{x, y\}$ whose endvertices are both vertices of $S^{\prime}$. Let also $G$ be the graph obtained from $G^{\prime}$ after the contraction of e to a vertex $v_{e}$, and let $S=S^{\prime} \backslash\{x, y\} \cup\left\{v_{e}\right\}$. Then $\left|\Pi_{G}(S)\right| \leq\left|\Pi_{G^{\prime}}\left(S^{\prime}\right)\right|$.

Proof. Similarly to the proof of Lemma 8.6, let us define an injection $i: \Pi_{G}(S) \hookrightarrow \Pi_{G^{\prime}}\left(S^{\prime}\right)$. Let $\mathcal{R} \in \Pi_{G}(S)$, and let $H$ be a subgraph of $G$ whose connected components define the packing $\mathcal{R}$ of $S$. We distinguish two cases. First, if $v_{e} \notin V(H)$, we define $i(\mathcal{R})$ to be the packing of $S^{\prime}$ given by the connected components of $H$. Otherwise, if $v_{e} \in V(H)$, let $H^{\prime} \subseteq G^{\prime}$ be the graph obtained from $H$ by removing $v_{e}$ and adding $x, y$, the edge $\{x, y\}$, and all the edges in $G^{\prime}$ between $x, y$ and the neighbors of $v_{e}$ in $H$. In this case we define $i(\mathcal{R})$ to be the packing of $S^{\prime}$ given by the connected components of $H^{\prime}$. It is again easy to check that if $\mathcal{R}_{1}, \mathcal{R}_{2} \in \Pi_{G}(S)$ with $\mathcal{R}_{1} \neq \mathcal{R}_{2}$, then $i\left(\mathcal{R}_{1}\right) \neq i\left(\mathcal{R}_{2}\right)$.

The following observation gives the obvious way to enumerate packings from partitions.

OBSERVATION 8.2. Let $G$ be a graph and let $S \subseteq V(G)$. Then $\Psi_{G}(S)=\bigcup_{S^{\prime} \subseteq S} \Pi_{G}\left(S^{\prime}\right)$.

Combining Lemma 8.5 and Observation 8.2 we obtain the following.

OBSERVATION 8.3. Let $G$ be a graph and let $S^{\prime} \subseteq S \subseteq V(G)$. Then $\left|\Psi_{G}\left(S^{\prime}\right)\right| \leq\left|\Psi_{G}(S)\right|$.

Let $H$ be a graph embedded in a surface $\Sigma$ with boundary. We denote by $\mathfrak{B}_{H}$ the collection of connected subsets of $\Sigma$ corresponding to the connected components of $H$. 
LEMMA 8.7. Let $G$ be a graph containing a set $A$ of vertices such that $G \backslash A$ is embedded in a surface $\Sigma$ with boundary. Let also $S$ be the set of vertices of $G$ that lie on the boundary of $\Sigma$. Then $\left|\Pi_{G}(S \cup A)\right| \leq\left|\Pi_{\Sigma^{A}}(S \cup A)\right|$.

PROOF. It is enough to prove that for every partition $\mathcal{R}$ in $\Pi_{G}(S \cup A)$ there is an extended partition family $\mathfrak{B}$ for $\left(\Sigma^{A}, S \cup A\right)$ such that $\mathcal{R}(\mathfrak{B})=\mathcal{R}$. For this, consider a subgraph $H$ of $G$ where $\mathcal{P}_{S \cup A}(H)=\mathcal{R}$. As $\mathcal{R} \in \Pi_{G}(S \cup A)$, it holds that $\cup \mathcal{R}=S \cup A$ and therefore $\cup \mathcal{R} \subseteq V(H)$. As $H \backslash A$ can be embedded in $\Sigma$, the set $\mathfrak{B}_{H \backslash A}$ is a partition family for $(\Sigma, S)$. Let now $H_{A}$ be the subgraph of $H$ formed by its edges that are not embedded in $\Sigma$. Observe that $H_{A}$ is isomorphic to a subgraph of $C_{A}$ and therefore its edges can be seen as a collection $\mathfrak{E}_{A}$ of flying edges where each apex vertex is contained in some edge of $\mathfrak{E}_{A}$. Let $\mathfrak{B}$ be the connected components of the $\operatorname{set} \cup\left(\mathfrak{B}_{H \backslash A} \cup \mathfrak{E}_{A}\right)$. Clearly, $\mathfrak{B}$ is an extended partition family for $\left(\Sigma^{A}, S \cup A\right)$. It is now easy to verify that $\mathcal{R}(\mathfrak{B})=\mathcal{R}$ and the lemma follows.

LEMMA 8.8. Let $G$ be a graph containing a set $A$ of vertices such that $G \backslash A$ is embedded in a surface $\Sigma$ with boundary. Let also $S$ be the set of vertices of $G$ that lie on the boundary of $\Sigma$ and $A^{\prime} \subseteq A$. Then, if $|S| \leq k$ and $|A|, \gamma(\Sigma), \beta(\Sigma) \leq \gamma$, then $\left|\Psi_{G}\left(S \cup A^{\prime}\right)\right|=\gamma^{O(\gamma)} \cdot k^{O(\gamma)} \cdot \gamma^{O(k)}$.

ProOF. From Observation 8.3, it is enough to prove the lemma for the case where $A^{\prime}=A$. From Lemmata 8.4 and 8.7, it follows that $\left|\Pi_{G}(S \cup A)\right|=\gamma^{O(\gamma)} \cdot k^{O(\gamma)} \cdot \gamma^{O(k)}$. From Lemma 8.5, we obtain that $\left|\Pi_{G}(W)\right| \leq\left|\Pi_{G}(S \cup A)\right|=\gamma^{O(\gamma)} \cdot k^{O(\gamma)} \cdot \gamma^{O(k)}$ for every $W \subseteq S \cup A$. Therefore, from Observation 8.2, $\left|\Psi_{G}(S \cup A)\right| \leq 2^{|S|+|A|} \cdot \gamma^{O(\gamma)} \cdot k^{O(\gamma)} \cdot \gamma^{O(k)}=\gamma^{O(\gamma)} \cdot k^{O(\gamma)} \cdot \gamma^{O(k)}$ and the lemma follows.

Let $\Sigma$ be a surface without boundary, and let $\mathcal{N}$ be a set of $O$-arcs in $\Sigma$ pairwise intersecting at zero-dimensional subsets of $\Sigma$. Then the closure of each connected component of $\Sigma \backslash \cup \mathcal{N}$ is called a pseudo-surface. Note that if the $O$-arcs are pairwise disjoint, then each pseudosurface is a surface with boundary. Note also that the boundary of a pseudo-surface is a subset of $\mathcal{N}$ and that the definition of the parameter $\theta(\mathcal{N})$ introduced in Section 6 can be naturally extended to pseudo-surfaces. If $\Sigma$ is a pseudo-surface with boundary given by a finite set $\mathcal{N}$ of $O$-arcs pairwise intersecting at finite zero-dimensional subsets of $\Sigma$, note that $\Sigma$ is a surface with boundary if and only if $\theta(\mathcal{N})=0$. Note also that the closure of each of the two connected components in the last condition of Definition 7.1 is a pseudo-surface.

LEMMA 8.9. Let $G$ be a graph embedded in a pseudo-surface $\Sigma$ whose boundary is given by a collection $\mathcal{N}$ of nooses of $G$ pairwise intersecting only at vertices of $G$, and such that $\theta(\mathcal{N})>0$. Let $S$ be the set of vertices of $G$ that lie on the boundary of $\Sigma$. Then there is a graph $G^{\prime}$ embedded in a pseudo-surface $\Sigma^{\prime}$ with boundary given by a collection $\mathcal{N}^{\prime}$ of nooses of $G^{\prime}$, such that

$-\theta\left(\mathcal{N}^{\prime}\right)=\theta(\mathcal{N})-1$

- $G$ is the result of the contraction of an edge in $G^{\prime}$;

- if $S^{\prime}$ is the set of vertices of $G^{\prime}$ that lie on the boundary of $\Sigma^{\prime}$, then $\left|S^{\prime}\right|=|S|+1$.

PROOF. Without loss of generality, let $v \in N_{1} \cap \ldots \cap N_{\ell}$, with $N_{1}, \ldots, N_{\ell} \in \mathcal{N}$ and $\ell \geq 2$, so by assumption $v \in S \subseteq V(G)$; for an illustration throughout the proof, see Figure 5 . We build from $\Sigma$ a pseudo-surface $\Sigma^{\prime}$ by replacing noose $N_{1}$ with a noose $N_{1}^{\prime}$ obtained from $N_{1}$ by slightly deforming it around $v$ in such a way that $v \notin N_{1}^{\prime}$ (note that this is clearly possible, as by assumption the nooses intersect only at vertices of $G$ ). As the nooses in $\Sigma$ and in $\Sigma^{\prime}$ intersect at the same vertices except for vertex $v$, we have that $\theta\left(\mathcal{N}^{\prime}\right)=\theta(\mathcal{N})-1$. We now construct $G^{\prime}$ from $G$ as follows: We start from the embedding of $G$ in $\Sigma$, and we embed it in $\Sigma^{\prime}$ in such a way that $v \in N_{2} \cap \ldots \cap N_{\ell}$. Finally, we add a new vertex $v^{\prime} \in N_{1}^{\prime}$ and we add the edge $\left\{v, v^{\prime}\right\}$. By construction, it is clear that $G$ can be obtained from $G^{\prime}$ by contracting edge $\left\{v, v^{\prime}\right\}$, and that $S^{\prime}=S \cup\left\{v^{\prime}\right\}$.

PROOF OF LEMMA 8.1:. In case $|\operatorname{mid}(e) \backslash A| \leq 2$, we have that $|\operatorname{mid}(e)|=O(\gamma)$ and the result follows as $\left|\Pi_{G_{e}}(\operatorname{mid}(e))\right|$ is upper-bounded by the $O(\gamma)$-th Bell number, and thus by $2^{O(\gamma \log \gamma)}$. In the remaining case, let $H$ be the graph of the polyhedral decomposition $(\mathcal{G}, A)$ of $G$ that corresponds to edge $e$. Let also $\mathcal{N}$ be the corresponding set of $O(\gamma)$ nooses meeting all vertices of $\operatorname{mid}(e) \backslash A$. Let also $\Sigma^{*}$ be the closure of the connected component of $\Sigma \backslash \bigcup_{N \in \mathcal{N}} N$ where the graph $G_{e} \backslash A$ is embedded. Clearly, $\Sigma^{*}$ is a pseudo-surface with boundary given by a set of nooses $\mathcal{N}$ with $\theta(\mathcal{N})=O(\gamma)$. By inductively applying Lemmata 8.6 and 8.9, we can assume that $\Sigma^{*}$ is a surface with boundary such that $O(|\operatorname{mid}(e)|+\gamma(\Sigma))=O(k+\gamma)$ of the vertices of $G_{e}$ lie on this boundary. Then the result follows directly from Lemma 8.8 by setting $G_{e}$ instead of $G, \Sigma^{*}$ instead of $\Sigma, A \cap \operatorname{mid}(e)$ instead of $A^{\prime}$, and $A \cap V\left(G_{e}\right)$ instead of $A$. 

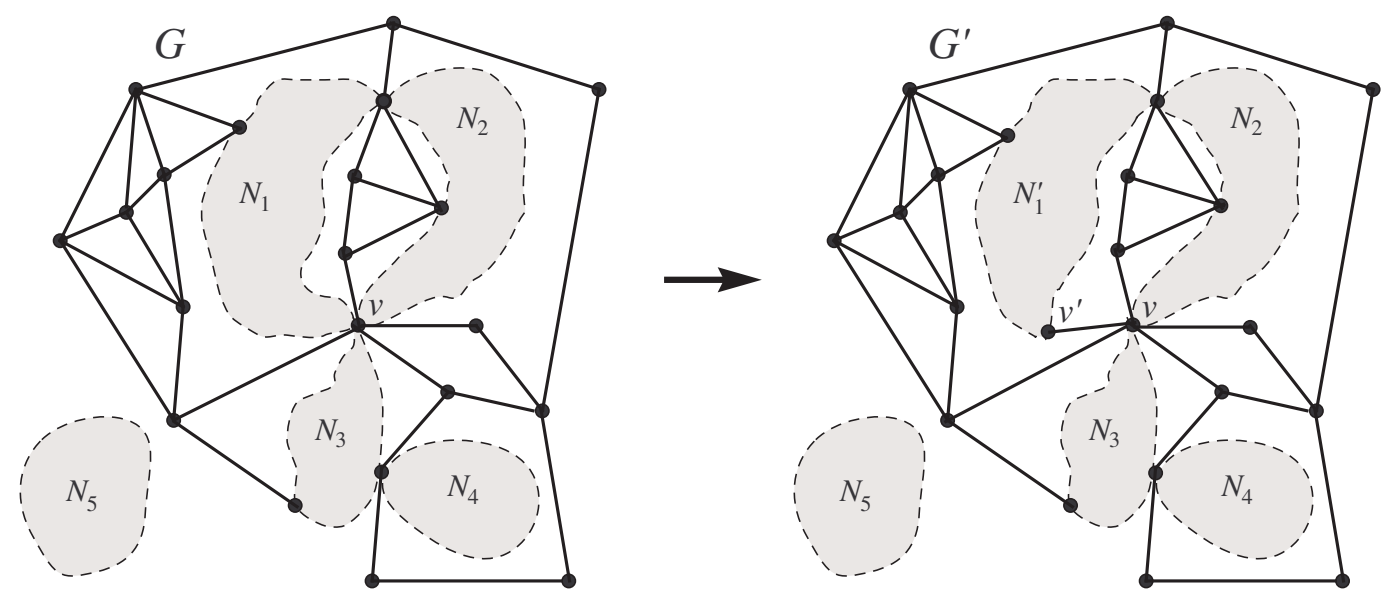

Fig. 5. Example of the construction of $\Sigma^{\prime}$ and $G^{\prime}$ in the proof of Lemma 8.9. On the left, we have a graph $G$ (depicted with thick lines) embedded in a pseudo-surface $\Sigma$ whose boundary is given by the set of nooses $\mathcal{N}=\left\{N_{1}, N_{2}, N_{3}, N_{4}, N_{5}\right\}$ (in grey) pairwise intersecting at vertices of $G$, with $\theta(\mathcal{N})=4$. On the right, the corresponding graph $G^{\prime}$ embedded in a pseudo-surface $\Sigma^{\prime}$ with boundary given by $\mathcal{N}^{\prime}=\left\{N_{1}^{\prime}, N_{2}, N_{3}, N_{4}, N_{5}\right\}$, and such that $\theta\left(\mathcal{N}^{\prime}\right)=3$. In this example, we have that $|S|=6$ and $\left|S^{\prime}\right|=7$.

\section{CONCLUSIONS AND OPEN PROBLEMS}

As stated in Theorem 3.2, our results can be summarized as follows: Every connected packingencodable problem whose input graph $G$ is embedded in a surface of Euler genus $\gamma$, and has branchwidth at most $k$, can be solved in $\gamma^{O(k)} \cdot k^{O(\gamma)} \cdot \gamma^{O(\gamma)} \cdot n^{O(1)}$ steps.

As we mentioned, the problems tackled in [Dorn et al. 2006] can be encoded with pairings, and therefore they can be seen as special cases of packing-encodable problems. As a result of this, we reproduce all the results of [Dorn et al. 2006]. Moreover, as our approach does not use planarization, our analysis provides algorithms where the dependence on the Euler genus $\gamma$ is better than the one in [Dorn et al. 2006]. In particular, the running time of the algorithms in [Dorn et al. 2006] is $2^{O\left(\gamma \cdot k+\gamma^{2} \cdot \log k\right)} \cdot n^{O(1)}$, while in our case the running time is $2^{O(\log \gamma \cdot k+\gamma \cdot \log k+\gamma \cdot \log \gamma)} \cdot n^{O(1)}$.

Dynamic programming is important for the design of subexponential exact or parameterized algorithms. Using the fact that bounded-genus graphs have branchwidth at most $O(\sqrt{\gamma \cdot n})$ [Fomin and Thilikos 2004], we derive the existence of exact algorithms in $O^{*}\left(2^{O(\log \gamma \cdot \sqrt{\gamma n}+\gamma \cdot \log n+\gamma \cdot \log \gamma}\right)$ steps for all connected packing-encodable problems. Moreover, using bidimensionality theory (see [Demaine et al. 2005; Demaine et al. 2006]), one can derive $2^{O(\gamma \cdot \log \gamma \cdot \sqrt{k}+\gamma \cdot \log k)} \cdot n^{O(1)}$ time parameterized algorithms for all bidimensional connected packing-encodable problems, where here $k$ is the corresponding parameter.

Note that the running time of our algorithms is conditioned by the construction of an appropriate surface cut decomposition. This preprocessing step takes $2^{4.5 k+O(\log k)} \cdot n^{3}$ steps by Theorem 7.2. Finding a preprocessing algorithm with better polynomial dependance remains open. As finding an optimal branch decomposition of a surface-embedded graph in polynomial time is open, it may be even possible that computing an optimal surface cut decomposition can be done in polynomial time.

Sometimes dynamic programming demands even more complicated encodings. We believe that our results can also serve in this direction. For instance, surface cut decompositions have recently been used in [Adler et al. 2010] for minor containment problems, where tables encode partitions of packings of the middle sets.

A natural extension of our results is to consider more general classes of graphs than bounded-genus graphs. This has been done in [Dorn et al. 2008] for problems where the tables of the algorithms encode pairings of the middle sets. Extending these results for connected packing-encodable problems (where tables encode subsets of the middle sets) using the planarization approach of [Dorn et al. 2008] appears to be a quite complicated task. We believe that our surface-oriented approach could be more successful in this direction and we find it an interesting, but non-trivial task [Rué et al. 2012]. 


\section{ACKNOWLEDGMENTS}

We would like to thank Sergio Cabello for inspiring discussions and for pointing us to several helpful topological references, and the anonymous referees for helpful comments that improved the presentation of the manuscript.

\section{References}

Adler, I., Dorn, F., Fomin, F. V., SAU, I., AND Thilikos, D. M. 2010. Faster Parameterized Algorithms for Minor Containment. In 12th Scandinavian Workshop on Algorithm Theory (SWAT). LNCS Series, vol. 6139. $322-333$.

AMIR, E. 2001. Efficient approximation for triangulation of minimum treewidth. In 17th Conference on Uncertainty in Artificial Intelligence (UAI). 7-15.

ARNBORG, S. 1985. Efficient algorithms for combinatorial problems on graphs with bounded decomposability - a survey. BIT 25, 1, 2-23.

ARvind, V. AND Mukhopadhyay, P. 2008. Derandomizing the isolation lemma and lower bounds for circuit size. In Proceedings of the 11th international workshop, APPROX 2008, and 12th international workshop, RANDOM 2008 on Approximation, Randomization and Combinatorial Optimization: Algorithms and Techniques. APPROX '08 / RANDOM '08. Springer-Verlag, Berlin, Heidelberg, 276-289.

BodlaEnder, H. L. 1988. Dynamic programming on graphs with bounded treewidth. In 15th International Colloquium on Automata, Languages and Programming (ICALP). LNCS Series, vol. 317. $105-118$.

CABEllo, S. AND MOHAR, B. 2007. Finding shortest non-separating and non-contractible cycles for topologically embedded graphs. Discrete and Computational Geometry 37, 213-235.

CAI, L. AND JUEDES, D. 2003. On the existence of subexponential parameterized algorithms. Journal of Computer and System Sciences 67, 4, $789-807$.

COURCELle, B. 1988. The monadic second-order logic of graphs: definable sets of finite graphs. In 14th International Workshop on Graph-theoretic Concepts in Computer Science (WG). LNCS Series, vol. 344. 30-53.

Cygan, M., Nederlof, J., Pilipczuk, M., Pilipczuk, M., van RooiJ, J. M. M., ANd Wojtaszczyk, J. O. 2011. Solving connectivity problems parameterized by treewidth in single exponential time. In IEEE 52nd Annual Symposium on Foundations of Computer Science, FOCS 2011, Palm Springs, CA, USA, October 22-25, 2011. 150-159.

Demaine, E. D., Fomin, F. V., Hajiaghayi, M. T., And Thilikos, D. M. 2005. Subexponential parameterized algorithms on graphs of bounded genus and $H$-minor-free graphs. Journal of the ACM 52, 6, 866-893.

Demaine, E. D., Hajiaghayi, M., And Thilikos, D. M. 2006. The Bidimensional Theory of Bounded-Genus Graphs. SIAM Journal on Discrete Mathematics 20, 2, 357-371.

Diestel, R. 2005. Graph Theory 3rd Ed. Graduate Texts in Mathematics Series, vol. 173. Springer Verlag.

Dorn, F., Fomin, F. V., AND Thilikos, D. M. 2006. Fast Subexponential Algorithm for Non-local Problems on Graphs of Bounded Genus. In 10th Scandinavian Workshop on Algorithm Theory (SWAT). LNCS Series, vol. 4059. 172-183.

Dorn, F., Fomin, F. V., AND Thilikos, D. M. 2007. Subexponential parameterized algorithms. In 34th International Colloquium on Automata, Languages and Programming (ICALP). LNCS Series, vol. $4596.15-27$.

Dorn, F., Fomin, F. V., AND Thilikos, D. M. 2008. Catalan structures and dynamic programming in $H$-minorfree graphs. In 19th annual ACM-SIAM Symposium on Discrete algorithms (SODA). Philadelphia, PA, USA, 631-640.

Dorn, F., PEnNinkx, E., BodlaEnder, H. L., AND Fomin, F. V. 2010. Efficient exact algorithms on planar graphs: Exploiting sphere cut decompositions. Algorithmica 58, 3, 790-810.

Downey, R. G. AND Fellows, M. R. 1999. Parameterized Complexity. Springer-Verlag, New York.

EPPSTEIN, D. 2003. Dynamic Generators of Topologically Embedded Graphs. In 14th annual ACM-SIAM Symposium on Discrete algorithms (SODA). 599-608.

Flajolet, P. AND Sedgewick, R. 2008. Analytic Combinatorics. Cambridge Univ. Press.

Flum, J. AND GROHE, M. 2006. Parameterized Complexity Theory. Springer.

Fomin, F. V. AND Thilikos, D. M. 2004. Fast Parameterized Algorithms for Graphs on Surfaces: Linear Kernel and Exponential Speed-Up. In 31st International Colloquium on Automata, Languages and Programming (ICALP). LNCS Series, vol. 3142. 581-592.

Fomin, F. V. And Thilikos, D. M. 2006. Dominating Sets in Planar Graphs: Branch-Width and Exponential SpeedUp. SIAM Journal on Computing 36, 2, 281-309.

Fomin, F. V. And Thilikos, D. M. 2007. On Self Duality of Pathwidth in Polyhedral Graph Embeddings. Journal of Graph Theory 55, 42-54.

Henzinger, M. R., RaO, S., And Gabow, H. N. 2000. Computing Vertex Connectivity: New Bounds from Old Techniques. Journal of Algorithms 34, 2, 222-250.

Impagliazzo, R., PATURI, R., AND ZANE, F. 2001. Which Problems Have Strongly Exponential Complexity? Journal of Computer and System Sciences 63, 4, 512-530. Special issue of FOCS 1998.

KAWARABAYAshi, K.-I., MohaR, B., AND REED, B. 2008. A simpler linear time algorithm for embedding graphs into an arbitrary surface and the genus of graphs of bounded tree-width. In Proceedings of the 200849 th Annual IEEE Symposium on Foundations of Computer Science. IEEE Computer Society, Washington, DC, USA, 771780 .

Lokshtanov, D., MARX, D., AND SAURABH, S. 2011a. Known algorithms on graphs of bounded treewidth are probably optimal. In 22st ACM-SIAM Symposium on Discrete Algorithms (SODA 2011). ACM-SIAM, San Francisco, California, 777-789.

Lokshtanov, D., MARX, D., AND SAURABH, S. 2011b. Slightly superexponential parameterized problems. In 22st ACM-SIAM Symposium on Discrete Algorithms (SODA 2011). 760-776. 
MoHAR, B. 1999. A linear time algorithm for embedding graphs in an arbitrary surface. SIAM J. Discrete Math. 12, 1, $6-26$.

Mohar, B. and Thomassen, C. 2001. Graphs on surfaces. John Hopkins University Press.

Mulmuley, K., VAZirani, U. V., AND VAZiRani, V. V. 1987. Matching is as easy as matrix inversion. In Proceedings of the nineteenth annual ACM symposium on Theory of computing. STOC '87. ACM, New York, NY, USA, 345354.

Niedermeier, R. 2006. Invitation to fixed-parameter algorithms. Oxford Lecture Series in Mathematics and its Applications Series, vol. 31. Oxford University Press, Oxford.

Robertson, N. AND Seymour, P. D. 1991. Graph minors. X. Obstructions to Tree-decomposition. J. Combin. Theory Series B 52, 2, 153-190.

Robertson, N. And Seymour, P. D. 1995. Graph minors. XII. Distance on a surface. J. Combin. Theory Series $B$ 64, 240-272.

Robertson, N. AND Seymour, P. D. 1999. Graph minors. XVI. Excluding a non-planar graph. J. Combin. Theory Series $B$ 77, 1-27.

RUÉ, J., SAU, I., AND Thilikos, D. M. 2011. Asymptotic enumeration of non-crossing partitions on surfaces. Manuscript submitted for publication, available at http://lanl.arxiv.org/abs/1104.2477.

RUÉ, J., SAU, I., AND THILIKoS, D. M. 2012. Dynamic programming for minor-free graphs. Manuscript in preparation.

SAU, I. And Thilikos, D. M. 2010. Subexponential Parameterized Algorithms for Degree-constrained Subgraph Problems on Planar Graphs. Journal of Discrete Algorithms 8, 3, 330-338.

Seymour, P. D. AND Thomas, R. 1994. Call routing and the ratcatcher. Combinatorica 14, 2, 217-241.

Telle, J. A. AND Proskurowski, A. 1997. Algorithms for vertex partitioning problems on partial $k$-trees. SIAM Journal on Discrete Mathematics 10, 4, 529-550.

Thomassen, C. 1989. The graph genus problem is NP-complete. Journal of Algorithms 10, 4, 568-576. 


\section{APPENDIX}

\section{Two examples of dynamic programming algorithms}

In this Appendix we present two examples of typical dynamic programming algorithms on graphs of bounded branchwidth. The first algorithm solves the VERTEX COVER problem, which is a problem whose solutions can be simply encoded by a subset of vertices. The second algorithm solves the CONNECTED VERTEX COVER problem, which is a packing-encodable problem, but is not known whether it can be encoded by either a subset or a pairing of vertices.

Dynamic programming for VERTEX COVER.. Given a graph $G$ and a non-negative integer $\ell$, we have to decide whether $G$ contains a set $S \subseteq V(G),|S| \leq \ell$, meeting all edges of $G$.

Let $G$ be a graph and $X, X^{\prime} \subseteq V(G)$ where $X \cap X^{\prime}=\emptyset$. We say that $\operatorname{vc}\left(G, X, X^{\prime}\right) \leq \ell$ if $G$ contains a vertex cover $S$ where $|S| \leq \ell$ and $X \subseteq S \subseteq V(G) \backslash X^{\prime}$. We assume that we are given a rooted branch decomposition $(T, \mu)$ of $G$ (see Section 3 for the definitions). Let $\mathcal{R}_{e}=\left\{(X, \ell) \mid X \subseteq \operatorname{mid}(e)\right.$ and $\left.\mathbf{v c}\left(G_{e}, X, \operatorname{mid}(e) \backslash X\right) \leq \ell\right\}$ and observe that $\mathbf{v c}(G) \leq \ell$ if and only if $(\emptyset, \ell) \in \mathcal{R}_{e_{r}}$. For each $e \in E(T)$ we can compute $\mathcal{R}_{e}$ by using the following dynamic programming formula:

$$
\mathcal{R}_{e}= \begin{cases}\{(X, \ell) \mid X \subseteq e \text { and } X \neq \emptyset \text { and } \ell \geq|X|\} & \text { if } e \in L(T) \\ \left\{(X, \ell) \mid \exists\left(X_{1}, \ell_{1}\right) \in \mathcal{R}_{e_{1}}, \exists\left(X_{2}, \ell_{2}\right) \in \mathcal{R}_{e_{2}}:\right. & \\ \left.\left(X_{1} \cup X_{2}\right) \cap \operatorname{mid}(e)=X \text { and } \ell_{1}+\ell_{2}-\left|X_{1} \cap X_{2}\right| \leq \ell\right\} & \text { if } e \notin L(T)\end{cases}
$$

Note that for each $e \in E(T),\left|\mathcal{R}_{e}\right| \leq 2^{|\operatorname{mid}(e)|} \cdot \ell$. Therefore, the above algorithm can check whether vc $(G) \leq \ell$ in $O\left(4^{\mathrm{bw}(G)} \cdot \ell^{2} \cdot|V(T)|\right)$ steps. Clearly, this simple algorithm is single-exponential in bw $(G)$. Moreover the above dynamic programming machinery can be adapted to many other combinatorial problems where the certificate of the solution is a (non-restricted) subset of vertices (e.g. Dominating SET, 3-Coloring, IndEPENDENT SET, among others).

Dynamic programming for CONnECTED VerTex COVER.. Suppose now that we are looking for a connected vertex cover of size $\leq \ell$. A simple application of the above dynamic programming formula does not work for this variant, as we should keep track of more information on $X$ towards encoding the connectivity demand.

Let $G$ be a graph, $X \subseteq V(G)$ and $\mathcal{H}$ be a (possibly empty) hypergraph whose vertex set is a subset of $X$, whose hyperedges are non-empty, pairwise non-intersecting, and such that each vertex of $\mathcal{H}$ belongs to some of its hyperedges (we call such a hypergraph partial packing of $X)$. Suppose that $\mathcal{H}$ is a partial packing of $\operatorname{mid}(e)$. We say that $\operatorname{cvc}\left(G_{e}, \mathcal{H}\right) \leq \ell$ if $G_{e}$ contains a vertex cover $S$ where $|S| \leq \ell$ and such that if $\mathcal{C}$ is the collection of the connected components of $G_{e}[S]$, then either $|E(\mathcal{H})|=|\mathcal{C}|$ and $(X,\{X \cap V(C) \mid C \in \mathcal{C}\})=\mathcal{H}$ or $E(\mathcal{H})=\emptyset$ and $|\mathcal{C}|=1$.

As before, let $\mathcal{Q}_{e}=\left\{(\mathcal{H}, \ell) \mid \operatorname{cvc}\left(G_{e}, \mathcal{H}\right) \leq \ell\right\}$ and observe that $\operatorname{cvc}(G) \leq \ell$ if and only if $((\emptyset, \emptyset), \ell) \in \mathcal{Q}_{e_{r}}$. The dynamic programming formula for computing $\mathcal{Q}_{e}$ for each $e \in E(T)$ is the following.

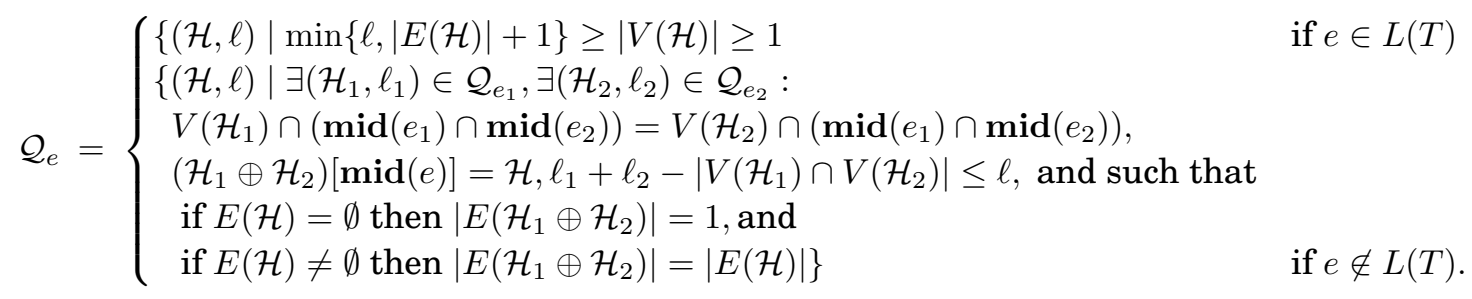

In the above formula, $\mathcal{H}_{1} \oplus \mathcal{H}_{2}$ is the hypergraph with vertex set $V\left(\mathcal{H}_{1}\right) \cup V\left(\mathcal{H}_{2}\right)$ where each of its hyperedges contains the vertices of each of the connected components of $\mathcal{H}_{1} \cup \mathcal{H}_{2}$. Note that in the case where $E(\mathcal{H})=\emptyset$, the current partial vertex cover must already be connected, which translates into $\left|E\left(\mathcal{H}_{1} \oplus \mathcal{H}_{2}\right)\right|=1$.

Clearly, each $\mathcal{H}$ corresponds to a collection of disjoint subsets of $X$ and the number of such collections for a given set $\operatorname{mid}(e)$ of $r$ elements is given by the $r$-th Bell number of $r$, denoted by $B_{r}$. By taking the straightforward upper bound $\left|B_{r}\right|=2^{O(r \log r)}$, we have that one can check whether an input graph $G$ has a connected vertex cover of size at most $\ell$ in $2^{O(\operatorname{bw}(G) \cdot \log \text { bw }(G))}$. $\ell \cdot|V(T)|$ steps.

As the growth of $B_{r}$ is not single-exponential, we cannot hope for a single-exponential (in bw $(G)$ ) running time using the above dynamic programming procedure. At this time, 
no deterministic algorithm is known for this problem running in time single-exponential in bw $(G)$. The same problem appears for numerous other problems where further restrictions apply to their solution certificates. Such problems can be connected variants of problems encodable by a subset of vertices, and others such as MAXIMUM INDUCED FOREST, MAXImum $d$-Degree-Bounded Connected Subgraph, Metric TSP, MaXimum $d$-DegreeBOUNDED CONNECTED INDUCED SUBGRAPH and all the variants studied in [Sau and Thilikos 2010], Connected Dominating Set, Connected $r$-Domination, FeEdback Vertex Set, Connected Feedback Vertex Set, Maximum Leaf Spanning Tree, Maximum Full-Degree Spanning Tree, Steiner Tree, or Maximum leaf Tree.

Received ; revised ; accepted 